Publication List

Juntendo Medical Journal

2021. $67(5), 473-493$

\title{
Publications from Juntendo University Graduate School of Medicine, 2019 [3/6]
}

\section{Neurosurgery}

〈Original Articles〉

1) Akiba C, Gyanwali B, Villaraza S, Nakajima M, Miyajima M, Cheng CY, Wong TY, Venketasubramanian N, Hilal S, Chen C: The prevalence and clinical associations of disproportionately enlarged subarachnoid space hydrocephalus (DESH), an imaging feature of idiopathic normal pressure hydrocephalus in community and memory clinic based Singaporean cohorts. J Neurol Sci. https:// doi.org/10.1016/j.jns.2019.1165102019.

2) Andica C, Hagiwara A, Hori M, Haruyama T, Fujita S, Maekawa T, Kamagata K, Yoshida MT, Suzuki M, Sugano H, Arai H, Aoki S: Aberrant myelination in patients with SturgeWeber syndrome analyzed using synthetic quantitative magnetic resonance imaging. Neuroradiology. 2019 Sep; 61: 1055-1066. doi: 10.1007/s00234-019-02250-9. Epub 2019 Jul 6. PMID:31280361.

3) Andica C, Hagiwara A, Hori M, Kamagata K, Koshino S, Maekawa T, Suzuki M, Fujiwara H, Ikeno M, Shimizu T, Suzuki H, Sugano H, Arai H, Aoki S: Review of Synthetic MRI in Pediatric brains: Basic Principle of MR Quantification, Its Features, Clinical Applications, and Limitations. J Neuroradiol. 2019 Mar 7. pii: S0150-9861 (17) 30488-1. doi: 10.1016/j.neurad. 2019.02.005. [Epub ahead of print]

4) Andica C, Kamagata K, Hatano T, Saito A, Uchida W, Ogawa T, Takeshige-Amano $\mathrm{H}$, Zalesky A, Wada A, Suzuki M, Hagiwara A, Irie R, Hori M, Kumamaru KK, Oyama G, Shimo Y, Umemura A, Pantelis C, Hattori N, Aoki S: Free-water imaging in white and gray matter in Parkinson's disease. Cells 2019, 8, 839; doi:10.3390/cells 8080839 .

An asterisk $(*)$ denotes doctoral works by Japanese students A dugger $(\dagger)$ denotes doctoral works by non-Japanese students.
5) Daida K, Nishioka K, Shimo Y, Umemura A, Yoshino H, Hattori N: Deep brain stimulation shows high efficacy in two patients with GCH1 variants. Parkinsonism Relat Disord. 2019; 65: 277-278.

6) de Vega S, Kondo A, Suzuki M, Arai H, Jiapaer S, Sabit H, Nakada M, Ikeuchi T, Ishijima M, Arikawa-Hirasawa E, Yamada Y, Okada Y: Fibulin-7 is overexpressed in glioblastomas and modulates glioblastoma neovascularization through interaction with angiopoietin-1. Int J Cancer. 2019 Oct15; 145: 2157-2169. doi: 10.1002/ijc.32306. Epub 2019 Apr 15. PMID: 30924128.

7) Doi K, Otani N, Hayashi M, Takeuchi S, Toyooka T, Wada K, Mori K: Mixed pial and dural arteriovenous fistula after craniotomy: case report and literature review. Br J Neurosurg. 2019; 9: 1-4.

8) Doi K, Otani N, Takeuchi S, Toyooka T, Wada $\mathrm{K}$, Mori K: A case of meningioma originating from the lateral wall of the cavernous sinus. Br J Neurosurg. 2019; 28: 1-3.

9) Doi K, Takeuchi S, Toyooka T, Otani N, Wada $\mathrm{K}$, Mori K: A case of trigeminal neuralgia caused by both duplicated superior cerebellar arteries. Neurol India. 2019; 67: 276-277.

10) Hara T, Iwamuro H, Ohara $Y$, Matsuoka $H$, Kikuchi N, Kimura T, Arai H, Mizuno J. Efficacy of Atlantoaxial Transarticular Screw Fixation Using Navigation-Guided Drill: Technical Note. World Neurosurg. 2019 Nov 4; 134: 378-382.

11) Hara T, Nakajima M, Sugano H, Karagiozov K, Miyajima M, Arai H: Cerebrospinal fluid over-drainage associated with upper cervical myelopathy: Successful treatment using a gravitational add-on valve in two cases. Interdisciplinary Neurosurgery: Advanced Tech-

This is a reprint of content originally published in Juntendo University HP. 
niques and Case Management 2019 (IF 0.34).

12) Hayakawa M, Sugiu K, Yoshimura S, Hishikawa T, Yamagami H, Fukuda-Doi M: Effectiveness of staged angioplasty for avoidanceof cerebral hyperperfusion syndrome after carotidrevascularization, Journal of Neurosurgery, 2019; 18: 1-11.

13) Hayashi K, Jin K, Nagamori C, Okanari K, Okanishi T, Honma Y, Iimura Y, Uda T, Takada L, Otsubo H: Sudden unexpected death in epilepsy in the bathtub. Epilepsy Behavior, 2019; 96, 33-40.

14) Hishii M, Matsumoto T, Arai H: Diagnosis and Treatment of Early-Stage Glioblastoma. Asian J Neurosurg. 2019 Apr-Jun; 14: 589-592. doi: 10.4103/ajns.AJNS_18_19.PMID:31143291.

15) Horikoshi $K$, Tsutsumi $S$, Ito $M$, Izumi $H$, Ishii $\mathrm{H}$ : Atypical spinal endodermal cyst presenting with aseptic meningitis. Radiol Case Rep 2019; 14: 561-4.

16) Iimura $Y$, Sugano $H$, Nakajima M, Higo $T$, Suzuki H, Mitsuhashi T, Ueda T, Karagiozov $\mathrm{K}$, Igarashi A, Otsubo H, Arai H: Is decremental modulation index on scalp EEG a sign of good seizure outcome? A Sturge-Weber syndrome case with epileptic spasms. Clin Neurophysiol. 2019 Sep; 130: 1499-1501. doi: 10.1016/j.clinph.2019.06.002. Epub 2019 Jun 22. No abstract available. PMID:31295718.

17) Ikawa F, Morita A, Tominari S, Nakayama T, Shiokawa Y, Date I, Nozaki K, Miyamoto S, Kayama T, Arai H: Rupture risk of small unruptured cerebral aneurysms. J Neurosurg. 2019 Jan 25:1-10. doi: 10.3171/2018.9. JNS181736.

18) Ikemura R, Tsutsumi S, Nonaka S, Okura H, Suzuki T, Ishii H: Pituitary adenoma presenting with intermittent oculomotor nerve paresis and unique MRI features. Radiol Case Rep 2019; 14: 1368-71.

19) Ikenouchi $Y$, Kamagata $K$, Andica $C$, Hatano T, Ogawa T, Takeshige-Amano H,Kamiya K, Wada A, Suzuki M, Fujita S, Hagiwara A, Irie R, Hori M, Oyama G, Shimo Y, Umemura A, Hattori N, Aoki S: Evaluation of white matter microstructure in patients with Parkinson's disease using microscopic fractional anisotropy. Neuroradiology. 2019 Nov 4. doi: 10. 1007/s00234-019-02301-1.
* 20) Iseki S, Mitome-Mishima Y, Suga Y, Yatomi K, Nonaka S, Miyamoto N, Yamamoto M, Arai H, Oishi H, Ogino I, Kondo A: Histological and transmission electron microscopy results after embolization with hydrosoft/ hydroframe coils in experimental swine aneurysm. BioMed Reserch International 2019.

21) Kada A, Ogasawara K, Kitazono T, Nishimura K, Sakai N, Onozuka D, Shiokawa Y, Miyachi S, Nagata I, Toyoda K, Hashimoto Y, Hasegawa Y, Hoshino H, Yoshimura S, Suzuki M, Tsujino A, Matsuda S, Kurogi R, Kurogi A, Ren N, Nishimura A, Arimura K, Hagihara A, Tominaga T, Kayama T, Arai H, Suzuki N, Miyamoto S, Ogawa A, Iihara K: J-ASPECT Study Collaborators. National trends in outcomes of ischemic stroke and prognostic influence of stroke center capability in Japan, 2010-2016. Int J Stroke. 2019 Oct 25: 1747493019884526. doi: 10.1177/174749301988 4526. [Epub ahead of print] PMID: 31653178.

22) Kumagai K, Mori K, Takeuchi S, Wada K: Surgical Training for the Management of Intraoperative Aneurysm Rupture Using a Three-Dimensional Artificial Model. Asian J Neurosurg. 2019; 14: 172-174.

23) Kumagai K, Tomiyama A, Takeuchi S, Otani N, Fujita M, Fujii K, Wada K, Mori K: New endovascular perforation subarachnoid hemorrhage model for investigating the mechanisms of delayed brain injury. J Neurosurg. 2019; 22: $1-11$.

24) Le Berre A, Kamagata K, Otsuka Y, Andica C, Hatano T, Saccenti L, Ogawa T, TakeshigeAmano H, Wada A, Suzuki M, Hagiwara A, Irie R, Hori M, Oyama G, Shimo Y, Umemura A, Hattori N, Aoki S: Convolutional neural network-based segmentation can help in assessing the substantia nigra in neuromelanin MRI. Neuroradiology. 2019; 61: 1387-1395.

25) Maehara T, Kamiya K, Fujimaki T, Matsumura A, Hongo K, Kuroda S, Matsumae M, Takeshima H, Sugo N, Nakao N, Saito N, Ikawa F, Tamura N, Sakurada K, Shimokawa S, Arai H, Tamura K, Sumita K, Hara S, Kato Y: Gender Equality Committee of the Japan Neurosurgical Society. A questionnaire to assess the challenges faced by women who quit working as full-time neurosurgeons. 
World Neurosurg. 2019 Aug 19. pii: S18788750 (19) 32200-4. doi: 10.1016/j.wneu.2019.08. 045. [Epub ahead of print] PMID:31437517.

* 26) Magami S, Miyamoto N, Ueno Y, Hira K, Tanaka R, Yamashiro K, Oishi H, Arai H, Urabe T, Hattori N: The Effects of astrocyte and oligodendrocyte lineage cell interaction on white matter injury under chronic cerebral hypoperfusion. Neuroscience, 201915 May; 406: 167-175.

27) Nakajima M, Hara T, Miyajima M, Akiba C, Kawamura K, Sugano H, Tange Y, Shimoji K, Karagiozov K, Arai H: Shunt malfunction and calcification of abdominal fascia tissue resulting in the obstruction of the abdominal catheter: case report. World Neurosurg. 2019 Mar 1. pii: S1878-8750 (19) 30404-8. doi: 10.1016/j. wneu.2019.01.285. [Epub ahead of print].

28) Nakajima M, Kuriyama N, Miyajima $M$, Ogino I, Akiba C, Kawamura K, Kurosawa M, Watanabe Y, Fukushima W, Mori E, Kato T, Sugano H, Tange Y, Karagiozov K, Arai H: Background Risk Factors Associated with Shunt Intervention for Possible Idiopathic Normal Pressure Hydrocephalus: A Nationwide Hospital-Based Survey in Japan. J Alzheimers Dis. 2019; 68: 735-744. doi: 10. 3233/JAD-180955.

29) Nishioka K, Suzuki M, Nakajima M, Hara T, Iseki M, Hattori N: Painful legs and moving toes syndrome evaluated through brain single photon emission computed tomography: a case series. J Neurol. 266: 717-725. doi: 10. 1007/s00415-019-09194-3. 2019 (IF 3.787).

30) Nishimura A, Nishimura K, Onozuka D, Matsuo R, Kada A, Kamitani S, Higashi T, Ogasawara K, Shimodozono M, Harada M, Hashimoto Y, Hirano T, Hoshino H, Itabashi R, Itoh Y, Iwama T, Kohriyama T, Matsumaru Y, Osato T, Sasaki M, Shiokawa Y, Shimizu H, Takekawa H, Nishi T, Uno M, Yagita Y, Ido K, Kurogi A, Kurogi R, Arimura K, Ren N, Hagihara A, Takizawa S, Arai H, Kitazono T, Miyamoto S, Minematsu K, Iihara K: J-ASPECT study collaborators. Development of Quality Indicators of Stroke Centers and Feasibility of Their Measurement Using a Nationwide Insurance Claims Database in Japan - J-ASPECT Study - Circ J. 2019 Oct
25; 83: 2292-2302. doi: 10.1253/circj.CJ-190089. Epub 2019 Sep 26. PMID: 31554766.

31) Oishi H, Fujii T, Suzuki M, Takano N, Teranishi K, Yatomi K, Kitamura T, Yamamoto M, Aoki S, Arai H: Usefulness of Silent MR Angiography for Intracranial Aneurysms Treated with a Flow-Diverter Device. AJNR Am J Neuroradiol. 2019 May; 40: 808-814. doi: 10. 3174/ajnr.A6047. Epub 2019 May 2. PMID: 31048297.

32) Otani N, Mori K, Wada K, Tomiyama A, Toyooka T, Takeuchi S, Nakao Y, Yamamoto $\mathrm{T}$, Arai H: Limited Indications for Clipping Surgery of Paraclinoid Aneurysm Based on Long-Term Visual Morbidity. World Neurosurg. 2019 Oct 16. pii: S1878-8750 (19) 32591-4. doi: 10.1016/j.wneu.2019.09.147. [Epub ahead of print] PMID: 31629143.

33) Saito A, Kamagata K, Ueda R, Nakazawa M, Andica C, Irie R, Nakajima M, Miyajima M, Hori M, Tanaka F, Arai H, Aoki S: Ventricular volumetry and free-water corrected diffusion tensor imaging of the anterior thalamic radiation in idiopathic normal pressure hydrocephalus. J Neuroradiol. 2019 Apr 26. pii: S0150-9861 (18) 30195-0. doi: 10.1016/j.neurad. 2019.04.003. [Epub ahead of print] PMID: 31034894.

34) Sato K, Aita N, Hokari Y, Kitahara E, Tani M, Izawa N, Hatori K, Nakamura R, Sasaki F, Sekimoto S, Jo T, Oyama G, Hatano T, Shimo Y, Iwamuro H, Umemura A, Hattori N, Fujiwara T: Balance and Gait Improvements of Postoperative Rehabilitation in Patients with Parkinson's Disease Treated with Subthalamic Nucleus Deep Brain Stimulation (STNDBS). Parkinsons Dis. 2019 Aug 4; 2019: 7104071. doi: 10.1155/2019/7104071.

35) Senda D, Orgun D, Shimizu A, Shimoji K, Miyajima M, Arai H, Mizuno H, Komuro Y: Quantitative Analysis of Change in Intracranial Volume After Posterior Cranial Vault Distraction and Frontal Orbital Advancement/Remodeling. J Craniofac Surg. 2019 Jan; 30: 23-27.

36) Suzuki H, Sugano H, Nakajima M, Higo T, Iimura Y, Mitsuhashi T, Fusegi K, Kakita A, Otsubo H, Arai H: The epileptogenic zone in pharmaco-resistant temporal lobe epilepsy 
with amygdala enlargement. Epileptic Disord. 2019 Jun 1; 21: 252-264. doi: 10.1684/epd.2019. 1075. PMID: 31225808.

37) Suzuki K, Yatomi K, Yamamoto M, Oishi H, Arai H: Endovascular therapy of distal anterior cerebral artery aneurysms: Single-institution clinical experience with 47 patients (49 aneurysms). Journal of Neuroendovascular Therapy. 2019; 13: 329-335.

38) Takagi T, Yoshimura S, Sakai N, Iihara K, Oishi H, Hirohata M, Matsumaru Y, Matsumoto Y, Yamagami H, Menon B, Almekhlafi M, Kamal N, Hill M, Goyal M: Distribution and current problems of acute endovascular therapy for large artery occlusion from a two-year national survey in Japan. Int J Stroke. 2019 Aug 14: 1747493019869706.

39) Teramoto S, Oishi H, Arai H: Comparative Analysis of Long-Term Effect of Stent-Assisted Coiling in Unruptured Sidewall-Type and Terminal-Type Aneurysms. World Neurosurg. 2019 Mar 7. pii: S1878-8750 (19) 30568-6. doi: 10.1016/j.wneu.2019.02.145. [Epub ahead of print].

40) Teramoto $\mathrm{S}$, Tange $\mathrm{Y}$, Ishii $\mathrm{H}$, Goto $\mathrm{H}$, Ogino I, Arai H: Mixed gangliocytoma-pituitary adenoma containing $\mathrm{GH}$ and GHRH $\mathrm{co}^{-}$secreting adenoma cells. Endocrinol Diabetes Metab Case Rep. 2019 Oct 3; 2019. pii: EDM 190099. doi: 10.1530/EDM-19-0099. [Epub ahead of print] PMID: 31581122.

41) Tomiyama A, Ichimura K: Signal transduction pathways and resistance to targeted therapies in glioma. Semin Cancer Biol. 2019 Oct; 58: 118-129.

42) Tomiyama A, Kobayashi $T$, Mori K, Ichimura K: Protein Phosphatases-A Touchy Enemy in the Battle Against Glioblastomas: A Review. Cancers (Basel). 2019 Feb 19; 11.

43) Toyooka T, Wada K, Otani N, Tomiyama A, Takeuchi S, Tomura S, Nishida S, Ueno H, Nakao Y, Yamamoto T, Mori K: Potential Risks and Limited Indications of the Supraorbital Keyhole Approach for Clipping Internal Carotid Artery Aneurysms. World Neurosurg X. 2019 Feb 26; 2.

44) Tsutsumi S, Oishi H, Nonaka S, Okura H, Suzuki T, Yasumoto Y, Ishii H: Orbital arteriovenous fistula coexistent with an arterio- venous hemangioma: a rare occurrence and review of literature. World Neurosurg 2019; 122: 287-92.

45) Tsutsumi S, Ono H, Ishii H, Yasumoto Y: An undescribed venous pathway intervening between the olfactory fossa and nasal vestibule. Surg Radiol Anat. 2019; 41: 485-90.

46) Tsutsumi S, Ono H, Ishii H, Yasumoto Y: Diploic veins of the cranial base: an anatomical study using magnetic resonance imaging. Surg Radiol Anat. 2019; 41: 1029-36.

47) Tsutsumi S, Ono H, Ishii H, Yasumoto Y: The intracanalicular segment of the hypoglossal nerve: an anatomical study using magnetic resonance imaging. J Clin Neurosci. 2019; 68: 295-301.

48) Tsutsumi S, Ono H, Ishii H, Yasumoto $\mathrm{Y}$ : Vertebral artery segment at the suboccipital dural penetration site: an anatomical study using magnetic resonance imaging. Childs Nerv Syst. 2019; 35: 683-7.

49) Tsutsumi S, Ono H, Ishii H, Yasumoto Y: Visualization of the supraorbital notch/ foramen using magnetic resonance imaging. J Clin Neurosci. 2019; 62: 212-5.

50) Tsutsumi S, Ono H, Ishii H, Yasumoto Y: Interdural high signal on CISS sequence: an alternative CSF pathway? Childs Nerv Syst 2019; 35: 487-91.

51) Tsutsumi S, Ono H, Yasumoto $Y$, Ishii H: Does the prone sleeping position affect the intracranial dural venous flow? Childs Nerv Syst 2019; 35: 913-6.

52) Tsutsumi S, Ono H, Yasumoto Y, Ishii H: Possible cerebrospinal fluid pathways in the middle fossa floor and pterional diploe: A magnetic resonance imaging study. Surg Radiol Anat. 2019; 41: 1045-51.

53) Tsutsumi $\mathrm{S}$, Ono H, Yasumoto $\mathrm{Y}$, Ishii H: The diaphragma sellae, diaphragm opening, and subdiaphragmatic cistern: an anatomical study using magnetic resonance imaging. Surg Radiol Anat. 2019; 41: 529-34.

54) Tsutsumi S, Ono H, Yasumoto Y, Ishii H: The superior ophthalmic vein: anatomical perspective for transvenous access to the cavernous sinus. J Craniofac Surg. 2019 in press.

55) Tsutsumi S, Ono H, Yasumoto $\mathrm{Y}$, Ishii H: Venous channels of the falx cerebri in adult 
Japanese population: Delineation using magnetic resonance imaging. Surg Radiol Anat. 2019; 41: 203-7.

56) Tsutsumi S: CT perfusion for identification of patients at risk for delayed cerebral ischemia during the acute phase after aneurysmal subarachnoid hemorrhage: a meta-analysis. Neurol India. 2019 in press.

57) Yamagami K, Kurogi R, Kurogi A, Nishimura K, Onozuka D, Ren N, Kada A, Nishimura A, Arimura K, Ido K, Mizoguchi M, Sakamoto T, Kayama T, Suzuki M, Arai H, Hagihara A, Iihara K: J-ASPECT Study Collaborators. The Influence of Age on the Outcomes of Traumatic Brain Injury: Findings from a Japanese Nationwide Survey (J-ASPECT Study-Traumatic Brain Injury). World Neurosurg. 2019 Oct; 130: e26-e46. doi: 10.1016/j. wneu.2019.05.140. Epub 2019 May 24.PMID: 31132488.

58) Yamamoto H, Hamasaki T, Onda K, Nakayama Y, Ishii A, Oishi H, Sakai N, Satow T: Evaluating the safety and technical effectiveness of a newly developed intravascular 'flow isolator' stent for the treatment of intracranial aneurysms: study protocol for a first-in-human single-arm multiple-site clinical trial in Japan. BMJ Open 2019; 9: e020966.

59) Yoshiura T, Wada K, Kumagai K, Takeuchi S, Toyooka T, Otani N, Mori K: Surgical Rescue Technique for Incomplete Burr Hole Using a Small Bone Piece and the Craniotome. World Neurosurg. 2019; 130: 106-109.

$\langle$ Reviews〉

1) Shimoji K, Hara T, Ohara Y: Controversies related to pediatric Chiari I malformation. Childs Nerv Syst. 2019; 35: 1695-1699.

2) Uchikado H, Nishimura Y, Hattori G, Morioka M, Ohara Y: Micro-anatomical structures of the lumbar intervertebral foramen for FESS. Review of the literatures. Journal of Spinal Surgery, 2019; doi: 10.21037/jss.2019.10.07.

〈Books〉

1) Iwamuro $H$, Shimo $Y$, Umemura $A$, Arai $H$, Hattori N: Local field potentials in the subthalamic nucleus during repetitive fist opening/ closing in Parkinson's disease patients. 13th International Basal Ganglia Society Meeting, Biarritz, FRANCE, May 1, 2019.
2) Wada K: Hyperbaric preconditioning-induced neuroprotection. In Hyperbaric Oxygen Therapy. Springer Nature Singapore. 2019; 43-52.

3) Shimoji K, Miyajima M, Cascone D, Cinalli G, Arai H: Hydrocephalus Associated with Cerebral Malformations in Pediatric Hydrocephalus, Editors: Cinalli, Giuseppe, Özek, M. Memet, Sainte-Rose, Christian (Eds.) Springer Nature Switzerland 2019.

\section{Anatomy and Life Structure}

〈Original Articles〉

1) Kawasaki Y, Matsumoto A, Miyaki T, Kinoshita M, Kakuta S, Sakai T, Ichimura K: Three-dimensional architecture of pericardial nephrocytes in Drosophila melanogaster revealed by FIB/SEM tomography. Cell Tissue Res. 2019; 378: 289-300.

2) Ichimura K, Miyaki T, Kawasaki Y, Kinoshita M, Kakuta S, Sakai T: Morphological processes of foot process effacement in puromycin aminonucleotide nephrosis revealed by FIB/ SEM tomography. J Am Soc Nephrol. 2019; 30: $96-108$.

\section{Organ and Cell Physiology}

〈Original Articles〉

1) Saito T, Kuma A, Sugiura $Y$, Ichimura $Y$, Obata M, Kitamura H, Okuda S, Lee HC, Ikeda K, Kanegae Y, Saito I, Auwerx J, Motohashi H, Suematsu M, Soga T, Yokomizo T, Waguri S, Mizushima N, Komatsu M: Autophagy regulates lipid metabolism through selective turnover of NCoR1, Nat Commun. 2019; 10: 1567.

2) Saito T, Kuma A, Sugiura $Y$, Ichimura $Y$, Obata M, Kitamura H, Okuda S, Lee H.C, Ikeda K, Kanegae Y, Saito I, Auwerx J, Motohashi H, Suematsu M, Soga T, Yokomizo T, Waguri S, Mizushima N, Komatsu M: Autophagy regulates lipid metabolism through selective turnover of NCoR1. Nat Commun. 2019; 10: 1567.

3) Tamura N, Kageyama S, Komatsu M, Waguri S: Hyperosmotic Stress Induces Unconventional Autophagy Independent of the Ulk1 Complex. Mol Cell Biol. 2019; 39. 


\section{Human Pathology}

〈Original Articles〉

1) Kodama M, Kobayashi D, Iihara K, Abe K, Sahara R, Sassa M, Yamada T, Furukawa S, Yao T, Uchida K, Tamura T, Gegi M, Eishi Y: Adenocarcinoma within anorectal fistulae: different clinicopathological characteristics between Crohn's disease- associated type and the usual type. Mod Pathol 2019; 32: 314-325.

2) Komori H, Ueyama H, Nagahara A, Akazawa Y, Takada T, Matsumoto K, Matsumoto K, Asaoka D, Hojo M, Yao T, Watanabe S: A prospective randomized trial of a potassium competitive acid blocker vs proton pump inhibitors on the effect of ulcer healing after endoscopic submucosal dissection of gastric neoplasia. J Int Med Res. 2019; 47: 1441-1452.

3) Ogawa H, Kaira K, Motegi Y, Yokobori T, Takada T, Katoh R, Osone K, Takahashi R, Katayama C, Oyama T, Kanai Y, Yao T, Asao T, Kuwano H, Shirabe K: Role of amino acid transporter expression as a prognostic marker in patients with surgically resected colorectal cancer. Anticancer Res. 2019; 39: 2535-2543.

4) Osone K, Yokobori T, Katayama C, Tahahashi R, Kato R, Tatsuki H, Tatada T, Yajima R, Motegi Y, Ogawa H, Fujii T, Ojima H, Nakamura J, Yao T, Shitabe K, Kuwano H: STMN1 accumulation is associated with dysplastic and neoplastic lesions in patients with ulcerative colitis. Oncology Letters. 2019; 18: 4712-4718.

5) Hasegawa N, Takeda Nakamura I, Ueno T, Kojima S, Kawazu M, Akaike K, Okubo T, Takagi T, Suehara Y, Hayashi T, Saito T, Kaneko K, Mano H, Kohsaka S: Detection of circulating sarcoma tumor cells using a microfluidic chip-type cell sorter. Sci Rep. 2019 Dec 27; 9: 20047.

6) Tsuyama S, Saito T, Akazawa Y, Yanai Y, Yatagai N, Akaike K, Hayashi T, Suehara Y, Takahashi F, Takamochi K, Hashimoto T, Kajiyama Y, Tsurumaru M, Fukunaga T, Yao T: Molecular and clinicopathological analyses of esophageal carcinosarcoma with special reference to morphological change. Virchows Arch. 2019 Oct; 475: 415-424.

7) Hidayat M, Mitsuishi Y, Takahashi F, Tajima
K, Yae T, Miyahara K, Hayakawa D, Winardi W, Ihara H, Koinuma Y, Wirawan A, Nurwidya F, Kato M, Kobayashi I, Sasaki S, Takamochi K, Hayashi T, Suehara Y, Moriyama M, Moriyama H, Habu S, Takahashi K: Role of FBXW7 in the quiescence of gefitinib-resistant lung cancer stem cells in EGFR-mutant non-small cell lung cancer. Bosn J Basic Med Sci. 2019 Nov 8; 19: 355-367.

8) Suehara Y, Alex D, Bowman AS, Middha S, Zehir A, Chakravarty D, Wang L, Jour G, Nafa K, Hayashi T, Jungbluth AA, Frosina D, Slotkin EK, Shukla NN, Meyers PA, Healey JH, Hameed M, Ladanyi M: Clinical genomic sequencing of pediatric and adult osteosarcoma reveals distinct molecular subsets with potentially targetable alterations. Clin Cancer Res. 2019 Nov 1; 25: 6346-6356.

9) Kohsaka S, Tatsuno K, Ueno T, Nagano M, Shinozaki-Ushiku A, Ushiku T, Takai D, Ikegami M, Kobayashi H, Kage H, Ando M, Hata K, Ueda H, Yamamoto S, Kojima S, Oseto K, Akaike K, Suehara Y, Hayashi T, Saito T, Takahashi F, Takahashi K, Takamochi K, Suzuki K, Nagayama S, Oda Y, Mimori K, Ishihara S, Yatomi Y, Nagase T, Nakajima J, Tanaka S, Fukayama M, Oda K, Nangaku M, Miyazono K, Miyagawa K, Aburatani H, Mano H: Comprehensive assay for the molecular profiling of cancer by target enrichment from formalin-fixed paraffin-embedded specimens. Cancer Sci. 2019 Apr; 110: 1464-1479. doi: 10.1111/cas.13968. Epub 2019 Mar 5.

10) Akazawa $Y$, Hayashi T, Saito T, Niwa K, Kamiyama H, Sasahara N, Sakamoto K, Nagahara A, Yao T: Histological characteristics of eosinophilic myenteric ganglionitis: an underrecognised cause of chronic intestinal pseudoobstruction. Virchows Arch. 2019 Mar; 474: 395-400. doi: 10.1007/s00428-018-02513-5.

11) Kato $S$, Hayashi $T$, Suehara $Y$, Hamanoue $H$, Yamanaka S, Ichikawa Y, Higurashi T, Ohashi K, Yamaguchi S, Nozaki Y, Terao Y, Saito T, Yao T, Nakajima A, Syed A, Zehir A, Ladanyi M, Kato S: Multicenter experience with large panel next-generation sequencing in patients with advanced solid cancers in Japan, Jpn J Clin Oncol. 2019 Feb 1; 49: 174-182.

12) Hattori $A$, Hirayama $S$, Matsunaga $T$, Hayashi 
T, Takamochi K, Oh S, Suzuki K: Distinct clinicopathological characteristics and prognosis based on the presence of ground glass opacity component in clinical-stage IA lung adenocarcinoma. J Thorac Oncol. 2019 Feb; 14: 265-275.

13) Nakanuma $Y$, Kakuda $Y$, Fukumura $Y$, Sugino T, Uesaka K, Serizawa M, Terada T, Ohnishi Y: The pathologic and genetic characteristics of the intestinal subtype of intraductal papillary neoplasms of the bile duct. Am J Surg Pathol. 2019; 43: 1212-1220.

14) Matsuda Y, Masuda $Y$, Shimoji K, Matsukawa M, Kinowaki Y, Fukumura Y, Kikuyama M, Arai T: Severe acute pancreatitis in autopsies associated with surgeries and severe inflammatory diseases. Pancreas 2019; 48: 1321-1328.

15) Sugimoto K, Ito T, Hulbert A, Chen C, Orita H, Maeda M, Moro H, Fukagawa T, Ushijima T, Katai H, Wada R, Sato K, Sakamoto K, Yu W, Considine M, Cope L, Brock MV: DNA methylation genome-wide analysis in remnant and primary gastric cancers. Gastric Cancer. 2019; 22: 1109-20.

16) Shimizu Y, Wakabayashi K, Hayashi Y, Hara K, Aoyama R, Niimi T, Tomino Y, Wada R, Hata M, Suzuki Y: MPGN Type 3 Associated with Pemphigus Herpetiformis Mimicking PGNMID and Dermatitis Herpetiformis. Case Rep Nephrol Dial. 21; 2019; 9: 15-24.

17) Ito $T$, Matoba $R$, Maekawa $H$, Sakurada $M$, Kushida T, Orita H, Wada R, Sato K: Detection of gene mutations in gastric cancer tissues using a commercial sequencing panel. Molecular and Clinical Oncology. 2019; 11: 455-460.

18) Asahina M, Saito T, Hayashi T, Fukumura $Y$, Mitani K, Yao T: Clinicopathological effect of PLAG1 fusion genes in pleomorphic adenoma and carcinoma ex pleomorphic adenoma with special emphasis on histological features. Histopathology. 2019; 74: 514-525. doi: 10.1111/ his.13759.

19) Akazawa $Y$, Hayashi T, Saito T, Niwa $K$, Kamiyama H, Sasahara N, Sakamoto K, Nagahara A, Yao T: Histological characteristics of eosinophilic myenteric ganglionitis, an underrecognised cause of chronic intestinal pseudo-obstruction. Virchows Arch. 2019 Jan 4. doi: 10.1007/s00428-018-02513-5.

20) Yatagai N, Saito T, Akazawa Y, Hayashi T, Yanai Y, Tsuyama S, Ueyama H, Murakami T, Watanabe S, Nagahara A, Yao T: Frequent loss of heterozygosity of SMAD4 locus and prognostic impacts of SMAD4 immunohistochemistry in gastric adenocarcinoma with enteroblastic differentiation. Human Pathol. 2019 Jun; 88: 18-26. doi: 10.1016/j.humpath. 2019.03.005. Epub 2019 Apr 1.

21) Yatagai N, Saito T, Akazawa $Y$, Hayashi T, Yanai Y, Tsuyama S, Ueyama H, Murakami T, Watanabe S, Nagahara A, Yao T: TP53 inactivation and expression of methylationassociated proteins in gastric adenocarcinoma with enteroblastic differentiation. Virchows Archiv. 2019 Mar; 474: 315-324. doi: 10.1007/ s00428-018-2508-9. Epub 2018 Dec 15.

22) Suehara Y, Mukaihara K, Okubo T, Kurihara T, Hayashi T, Kohsaka S, Kazuno S, Keisuke Akaike K, Kim Y, Kaneko K, Saito T: Protein Expression Profiles Corresponding to Histological Changes with Denosumab Treatment in Giant Cell Tumors of Bone. PROTEOMICS - Clinical Applications. 2019 Jul 9: e1800147. doi: 10.1002 prca.201800147.

23) Tsuyama S, Saito T, Akazawa Y, Yanai Y, Akaike K, Hayashi T, Suehara Y, Takahashi F, Takamochi K, Hashimoto T, Kajiyama Y, Tsurumaru M, Fukunaga T, Takashi Yao T: Molecular and clinicopathological analyses in esophageal carcinosarcoma with special reference on the differentiation mechanism. Virchows Archiv. 2019 Oct; 475: 415-424. doi: 10.1007/ s00428-019-02643-4. Epub 2019 Aug 23.

24) Inoue S, Hirota $Y$, Ueno T, Fukui $Y$, Yoshida E, Hayashi T, Kojima S, Takeyama R, Hashimoto T, Kiyono T, Taguchi A, Tanaka T, Tanaka Y, Ikemura M, Sakata S, Takeuchi K, Muraoka A, Osuka S, Saito T, Oda K, Osuga Y, Terao Y, Kawazu M, Mano H: Uterine adenomyosis is an oligoclonal disorder associated with KRAS mutations. Nat Comm. 2019 Dec 19; 10: 5785. doi: 10.1038/s41467019-13708-y.

〈Books〉

1) Yao T, Wada R: Pathology of gastric cancer. In: Shiotani A. ed. Gastric cancer. With special focus on studies from Japan. Singapore: 
Springer Nature Singapore. 2019; 63-68.

2) Yao T, Wada R: Pathology of Gastric Cancer. Gastric Cancer.Edited by Shiotani A, Springer, Singapore, 2019; 63-75.

\section{Cellular and Molecular Pharmacology}

〈Original Articles〉

1) Eshima H, Tamura $Y$, Kakehi S, Nakamura K, Kurebayashi N, Murayama T, Kakigi R, Sakurai T, Kawamori R, Watada H: Dysfunction of muscle contraction with impaired intracellular $\mathrm{Ca}^{2+}$ handling in skeletal muscle and the effect of exercise training in male $\mathrm{db} / \mathrm{db}$ mice. J Appl Physiol. 2019; 126: 170-182.

2) Numata-Uematsu Y, Wakatsuki S, Nagano S, Shibata M, Sakai K, Ichinohe N, Mikoshiba K, Ohshima T, Yamashita N, Goshima Y, Araki $\mathrm{T}$ : Inhibition of collapsin response mediator protein-2 phosphorylation ameliorates motor phenotype of ALS model mice expressing SOD1G93A. Neurosci Res. 2019; 139: 63-68.

3) Saeki T, Kimura T, Hashidume K, Murayama T, Yamamura H, Ohya S, Suzuki Y, Nakayama $\mathrm{S}$, Imaizumi Y: Conversion of $\mathrm{Ca}^{2+}$ oscillation into propagative electrical signals by $\mathrm{Ca}^{2+}-$ activated ion channels and connexin as a reconstituted $\mathrm{Ca}^{2+}$ clock model for the pacemaker activity. Biochem Biophys Res Commun, 2019; 510: 242-247.

4) Suzuki C, Tanida I, Ohmuraya M, Oliva Trejo JA, Kakuta S, Sunabori T, Uchiyama Y: Lack of Cathepsin D in the Renal Proximal Tubular Cells Resulted in Increased Sensitivity against Renal Ischemia/Reperfusion Injury. Int J Mol Sci. 2019; 20: e1711.

5) Sou YS, Kakuta S, Kamikubo Y, Niisato K, Sakurai T, Parajuli LK, Tanida I, Saito H, Suzuki N, Sakimura K, Maeda Y, Kinoshita T, Uchiyama Y, Koike M: Cerebellar neurodegeneration and neuronal circuit remodeling in Golgi $\mathrm{pH}$ regulator-deficient mice. eNeuro, 2019; 6: e0427-18.2019.

6) Uzu M, Nonaka M, Miyano K, Sato H, Kurebayashi N, Yanagihara K, Sakurai T, Hisaka A, Uezono Y: A novel strategy for treatment of cancer cachexia targeting xanthine oxidase in the brain. J Pharmacol Sci. 2019; 140: 109-112.

7) Narita K, Suzuki N, Himi N, Murayama T, Nakagawa T, Okabe N, Nakamura-Maruyama
E, Hayashi N, Sakamoto I, Miyamoto O, Kuba $\mathrm{K}$ : Effects of intravesicular loading of a $\mathrm{Ca}^{2+}$ chelator and depolymerization of actin fibers on neurotransmitter release in frog motor nerve terminals. Eur J Neurosci. 2019; 50: 1700-1711.

8) Mori S, Iinuma $H$, Manaka N, Ishigami-Yuasa M, Murayama T, Nishijima Y, Sakurai A, Arai R, Kurebayashi N, Sakurai T, Kagechika H: Structural development of a type-1 ryanodine receptor (RyR1) $\mathrm{Ca}^{2+}$-release channel inhibitor guided by endoplasmic reticulum $\mathrm{Ca}^{2+}$ assay. Eur J Med Chem. 2019; 179: 837848.

9) Murayama T, Kurebayashi N: Assays for Modulators of Ryanodine Receptor (RyR)/ $\mathrm{Ca}^{2+}$ Release Channel Activity for Drug Discovery for Skeletal Muscle and Heart Diseases. Curr Protoc Pharmacol. 2019; 87: e71.

10) Suzuki C, Tanida I, Oliva Trejo JA, Kakuta S, Uchiyama Y: Autophagy Deficiency in Renal Proximal Tubular Cells Leads to an Increase in Cellular Injury and Apoptosis under Normal Fed Conditions. Int J Mol Sci. 2019; 21: e155.

11) Fukunaga I, Fujimoto A, Hatakeyama K, Kurebayashi N, Ikeda K, Kamiya K: Generation of Functional CX26-Gap-Junction-PlaqueForming Cells with Spontaneous $\mathrm{Ca}^{2+}$ Transients via a Gap Junction Characteristic of Developing Cochlea. Curr Protoc Stem Cell Biol. 2019; 51: e100.

$\langle$ Reviews〉

1) Yamashita N: Retrograde signaling via axonal transport through signaling endosomes. J Pharmacol Sci. 2019; 141: 91-96.

〈Books〉

1) Kamikubo Y, Sakurai T: Co-immunoprecipitation Analysis of GPCR Complexes in the Central Nervous System. Co-Immunoprecipitation Methods for Brain Tissue, Springer. 2019; 49-64.

\section{Gastroenterology}

〈Original Articles〉

1) Takeda T, Asaoka D, Nojiri S, Nishiyama M, Ikeda A, Yatagai N, Ishizuka K, Hiromoto T, Okubo S, Suzuki M, Nakajima A, Nakatsu Y, Komori H, Akazawa Y, Nakagawa Y, Izumi 
K, Matsumoto K, Ueyama H, Sasaki H, Shimada Y, Matsumoto K, Osada T, Hojo M, Kato M, Nagahara A: Linked Color Imaging and the Kyoto Classification of Gastritis: Evaluation of Visibility and Inter-Rater Reliability. Digestion, 2019; 12: 1-10.

2) Asaoka D, Takeda T, Sasaki H, Shimada Y, Matsumoto K, Ueyama H, Matsumoto K, Izumi K, Komori H, Akazawa Y, Osada T, Hojo M, Nagahara A: Evaluation of symptomatic reflux esophagitis in proton pump inhibitor users. Biomed Rep. 2019 May; 10: 277-282.

3) Komori H, Ueyama H, Nagahara A, Akazawa Y, Takeda T, Matsumoto K, Matsumoto K, Asaoka D, Hojo M, Yao T, Watanabe S: A prospective randomized trial of a potassium competitive acid blocker vs proton pump inhibitors on the effect of ulcer healing after endoscopic submucosal dissection of gastric neoplasia. J Int Med Res. 2019; 47: 1441-1452.

4) Atsukawa M, Tsubota A, Toyoda H, Takaguchi K, Nakamuta M, Watanabe T, Michitaka K, Ikegami T, Nozaki A, Uojima H, Fukunishi S, Genda T, Abe H, Hotta N, Tsuji K, Ogawa C, Tachi Y, Shima T, Shimada N, Kondo C, Akahane T, Aizawa Y, Tanaka Y, Kumada T, Iwakiri K: The efficacy and safety of glecaprevir plus pibrentasvir in 141 patients with severe renal impairment: a prospective, multicenter study. Aliment Pharmacol Ther. 2019 May; 49: 1230-1241.

5) Yoshida M, Takizawa K, Nonaka S, Shichijo S, Suzuki S, Sato C5, Komori H, Minagawa T, Oda I2, Uedo N, Hirasawa K, Matsumoto K, Sumiyoshi T, Mori K, Gotoda T, Ono H1; CONNECT-E Study Group: Conventional versus traction-assisted endoscopic submucosal dissection for large esophageal cancers: a multicenter, randomized controlled trial (with video). Gastrointest Endosc. 2020 Jan; 91: 55-65. e2. doi: 10.1016/j.gie.2019.08.014. Epub 2019 Aug 21.

6) Shibuya T, Nomura K, Okahara K, Haga K, Nomura O, Murakami T, Uchida S, Kodani T, Ishikawa D, Sakamoto N, Ogihara T, Osada T, Nagahara A: Budesonide Foam for Ulcerative Colitis Patients Experiencing Inadequate Response to Biological Therapy. Med Sci Monit. 2019 Dec 22; 25: 9855-9863.
7) Motoya S, Tanaka H, Shibuya T, Osada T, Yamamoto T, Hongo H, Mizuno C, Saito D, Aoyama N, Kobayashi T, Ito H, Tanida S, Nojima M, Kokuma S, Hosoi E: Safety and effectiveness of granulocyte and monocyte adsorptive apheresis in patients with inflammatory bowel disease in special situations: a multicentre cohort study. BMC Gastroenterol. 2019 Nov 21; 19: 196.

9) Takahashi M, Ishikawa D, Sasaki T, Lu YJ, Kuwahara-Arai K, Kamei M, Shibuya T, Osada T, Hiramatsu K, Nagahara A: Faecal freezing preservation period influences colonization ability for faecal microbiota transplantation. J Appl Microbiol. 2019 Mar; 126: 973-984.

* 10) Suzuki M, Kon K, Ikejima K, Arai K, Uchiyama A, Aoyama T, Yamashina S, Ueno T, Watanabe S: The chemical chaperone 4-phenylbutyric acid prevents alcohol-induced liver injury in obese KK-Ay mice. Alcohol Clin Exp Res. 2019; 43: 617-627.

* 11) Kitagawa R, Kon K, Uchiyama A, Arai K, Yamashina S, Kuwahara-Arai K, Kirikae T, Ueno T, Ikejima K: Rifaximin prevents ethanol-induced liver injury in obese $\mathrm{KK}-\mathrm{Ay}$ mice through modulation of small intestinal microbiota signature. Am J Physiol Gastrointest Liver Physiol. 2019; 317: G707-G715.

12) Sato T, Yamashina S, Izumi K, Ueno T, Koike M, Ikejima K, Peters C, Watanabe S: Cathepsin L-deficiency enhances liver regeneration after partial hepatectomy. Life Sci. 2019; 221: 293300.

13) Moniaga CS, Iwamoto S, Kitamura T, Fujishiro M, Takahashi N, Katsunari K, Ogawa H, Tominaga M, Takamori K: Plasma Dynorphin A Concentration Reflects the Degree of Pruritus in Chronic Liver Disease: A Preliminary Report. Acta Derm Venereol. 2019 Apr 1; 99: 442-443.

14) Shimada $Y$, Matsumoto K, Ikeda Y, Amano N, Sato S, Murata A, Tomishima K, Tsuzura H, Sato S, Iijima K, Nagahara A, Genda T: Does PPI play a protective role in gastric mucosa in patients taking doacs? and also in patients taking vka? Gastroenterology, 2019; 156: S503-S504.

15) Shimada Y, Matsumoto K, Ikeda Y, Amano 
N, Sato S, Murata A, Tomishima K, Tsuzura H, Sato S, Iijima K, Nagahara A, Genda T: Influence of clostridium butyricum as probiotics supplementation on helicobacter pylori eradication. Gastroenterology, 2019; 156: S531S531.

16) Mori H, Suzuki H, Omata F, Masaoka T, Asaoka D, Kawakami K, Mizuno S, Kurihara N, Nagahara A, Sakaki N, Ito M, Kawamura Y, Suzuki M, Shimada Y, Sasaki H, Matsuhisa T, Torii A, Nishizawa T, Mine T, Ohkusa T, Kawai T, Tokunaga K,Takahashi S: Current status of first- and second-line Helicobacter pylori eradication therapy in the metropolitan area: a multicenter study with a large number of patients. Therap Adv Gastroenterol. 2019; 12.

17) Tsuzura H, Ikeda $Y$, Hayashida S, Takahashi S, Amano N, Sato S, Murata A, Sato S, Shimada Y, Iijima K, Genda T: Super paramagnetic iron oxide magnetic resonance imaging (SPIO-MRI) shows impaired phagocytic function of kupffer cells in severe alcoholic hepatitis. Hepatology. 2019; 70: 850A851A.

18) Akazawa Y, Hayashi T, Saito T, Niwa K, Kamiyama H, Sasahara N, Sakamoto K, Nagahara A, Yao T: Histological characteristics of eosinophilic myenteric ganglionitis, an underrecognised cause of chronic intestinal pseudo-obstruction. Virchows Archiv, 2019; 474: 395-400.

19) Yatagai N, Saito $T$, Akazawa $Y$, Hayashi T,Yanai Y, Tsuyama S, Murakami T, Ueyama H,Watanabe S, Nagahara A, Yao T: Frequent loss of heterozygosity of SMAD4 locus and prognostic impacts of SMAD4 immunohistochemistry in gastric adenocarcinoma with enteroblastic differentiation. Hum Pathol. 2019 Jun; 88: 18-26.

20) Yatagai N, Saito T,Akazawa $Y$, Hayashi T, Yanai Y, Tsuyama S, Ueyama H, Murakami T, Watanabe S, Nagahara A, Yao T: TP53 inactivation and expression of methylationassociated proteins in gastric adenocarcinoma with enteroblastic differentiation. Virchows Arch. 2019 Mar; 474: 315-324.

21) Sagawa E, Okubo H, Ando H, Sorin Y, Kanazawa R, Nakadera E, Fukada H, Kokubu
S, Miyazaki A: Plasma concentration and efficacy of tolvaptan in cirrhotic patients with refractory ascites. J Pharmacol Sci. 2019 Feb 27. 2019; 139: 373-376.

22) Toyoda H, Atsukawa M, Uojima H, Nozaki A, Tamai H, Takaguchi K, Fujioka S, Nakamuta M, Tada T, Yasuda S, Chuma M, Senoh T, Tsutsui A, Yamashita N, Hiraoka A, Michitaka K, Shima T, Akahane T, Itobayashi E, Watanabe T, Ikeda H, Iio E, Fukunishi S, Asano T, Tachi Y, Ikegami T, Tsuji K, Abe H, Kato K, Mikami S, Okubo H, Shimada N, Ishikawa T, Matsumoto Y, Itokawa N, Arai T, Tsubota A, Iwakiri K, Tanaka Y, Kumada $\mathrm{T}$ : Trends and Efficacy of Interferon-Free Anti-hepatitis C Virus Therapy in the Region of High Prevalence of Elderly Patients, Cirrhosis, and Hepatocellular Carcinoma: A Real-World, Nationwide, Multicenter Study of 10688 Patients in Japan. Open Forum Infect Dis. 2019 Apr 15; 6: ofz185.

23) Takaguchi K, Toyoda H, Tsutsui A, Suzuki $\mathrm{Y}$, Nakamuta $\mathrm{M}$, Imamura $\mathrm{M}$, Senoh $\mathrm{T}$, Nagano T, Tada T, Tachi Y, Hiraoka A, Michitaka K, Shibata H, Joko K, Okubo H, Tsuji K, Takaki S, Watanabe T, Ogawa C, Chayama K, Kumada T, Kudo M, Kumada H: Real-world virological efficacy and safety of daclatasvir/asunaprevir/beclabuvir in patients with chronic hepatitis $\mathrm{C}$ virus genotype 1 infection in Japan. J Gastroenterol. 2019 Aug; 54: 742-751.

24) Toyoda H, Atsukawa M, Watanabe T, Nakamuta M, Uojima H, Nozaki A, Takaguchi K, Fujioka S, Iio E, Shima T, Akahane T, Fukunishi S, Asano T, Michitaka K, Tsuji K, Abe H, Mikami S, Okubo H, Okubo T, Shimada N, Ishikawa T, Moriya A, Tani J, Morishita A, Ogawa C, Tachi Y, Ikeda H, Yamashita N, Yasuda S, Chuma M, Tsutsui A, Hiraoka A, Ikegami T, Genda T, Tsubota A, Masaki T, Tanaka Y, Iwakiri K, Kumada T: Real-world experience of 12 -week DAA regimen of glecaprevir and pibrentasvir in patients with chronic HCV infection. J Gastroenterol Hepatol. 2019 Oct 14. doi: 10.1111/ jgh.14874. [Epub ahead of print].

25) Ikeda H, Watanabe T, Atsukawa M, Toyoda H, Takaguchi K, Nakamuta M, Matsumoto N, 
Okuse C, Tada T, Tsutsui A, Yamashita N, Kondo C, Hayama K, Kato K, Itokawa N, Arai T, Shimada N, Asano T, Uojima H, Ogawa C, Mikami S, Ikegami T, Fukunishi S, Asai A, Iio E, Tsubota A, Hiraoka A, Nozaki A, Okubo H, Tachi Y, Moriya A, Oikawa T, Matsumoto Y, Tsuruoka S, Tani J, Kikuchi K, Iwakiri K, Tanaka Y, Kumada T: Evaluation of 8-week glecaprevir/pibrentasvir treatment in directacting antiviral-naïve noncirrhotic HCV genotype 1 and 2infected patients in a real-world setting in Japan. J Viral Hepat. 2019 Nov; 26: 1266-1275.

26) Atsukawa M, Tsubota A, Takaguchi K, Toyoda H, Iwasa M, Ikegami T, Chuma M, Nozaki A, Uojima H, Hiraoka A, Fukunishi S, Yokohama K, Tada T, Kato K, Abe H, Tani J, Okubo H, Watanabe T, Hattori N, Tsutsui A, Senoh T, Yoshida Y, Okubo T, Itokawa N, Nakagawa-Iwashita A, Kondo C, Arai T, Michitaka K, Iio E, Kumada T, Tanaka Y, Takei Y, Iwakiri K: Analysis of factors associated with the prognosis of cirrhotic patients who were treated with tolvaptan for hepatic edema. J Gastroenterol Hepatol. 2019 Dec 27. doi: 10. 1111/jgh.14965. [Epub ahead of print].

28) Mizuno S, Nakai $Y$, Isayama $H$, Yoshikawa T, Saito K, Takahara N, Kogure H, Tada M, Hayashi N, Koike K: Visceral adiposity and high adiponectin levels are associated with the prevalence of pancreatic cystic lesions. Int J Obes (Lond), 2019; 43: 169-175.

29) Hakuta R, Kawahata S, Kogure H, Nakai $Y$, Saito K, Saito T, Hamada T, Takahara N, Uchino R, Mizuno S, Tsujino T, Tada M, Sakamoto N, Isayama H, Koike K: Endoscopic papillary large balloon dilation and endoscopic papillary balloon dilation both without sphincterotomy for removal of large bile duct stones: A propensity-matched analysis. Dig Endosc. 2019; 31: 59-68.

30) Tomita M, Saito S, Makimoto S, Yoshida S, Isayama H, Yamada T, Matsuzawa T, Enomoto T, Kyo R, Kuwai T, Hirata N, Shimada M, Hirakawa T, Koizumi K, Saida Y: Self-expandable metallic stenting as a bridge to surgery for malignant colorectal obstruction: pooled analysis of 426 patients from two prospective multicenter series. Surg Endosc.
2019; 33: 499-509.

31) Kuwai $T$, Yamaguchi T, Imagawa H, Yoshida S, Isayama H, Matsuzawa T, Yamada T, Saito S, Shimada M, Hirata N, Sasaki T, Koizumi K, Maetani I, Saida Y: Factors related to difficult self-expandable metallic stent placement for malignant colonic obstruction: A post-hoc analysis of a multicenter study across Japan. Dig Endosc, 2019; 31: 51-58.

32) Hakuta R, Hamada T, Nakai Y, Isayama $H$, Kogure H, Takahara N, Mizuno S, Yagioka H, Togawa O, Matsubara S, Ito Y, Yamamoto N, Tada M, Koike K: Early pancreatic stent placement in wire-guided biliary cannulation: A multicenter retrospective study. J Gastroenterol Hepatol. 2019; 34: 1116-1122.

33) Nakai Y, Isayama H, Kawakami H, Ishiwatari H, Kitano M, Ito Y, Yasuda I, Kato H, Matsubara $\mathrm{S}$, Irisawa A, Itoi T: Prospective multicenter study of primary EUS-guided choledochoduodenostomy using a covered metal stent. Endosc Ultrasound. 2019; 8: 111-117.

34) Saito K, Isayama H, Nakai Y, Takahara N, Ishigaki K, Takeda T, Hakuta R, Saito T, Uchino R, Kishikawa T, Hamada T, Mizuno S, Sasaki T, Kogure H, Matsubara S, Yamamoto N, Ijichi H, Tateishi K, Tada M, Koike K: A phase II trial of gemcitabine, S-1 and LV combination (GSL) therapy in patients with advanced pancreatic cancer. Invest New Drugs. 2019; 37: 338-344.

35) Nakai Y, Kogure H, Isayama H, Koike K: Endoscopic Ultrasound-Guided Biliary Drainage for Unresectable Hilar Malignant Biliary Obstruction. Clin Endosc. 2019; 52: 220-225.

36) Kamisawa T, Nakazawa T, Tazuma S, Zen Y, Tanaka A, Ohara H, Muraki T, Inui K, Inoue D, Nishino T, Naitoh I, Itoi T, Notohara K, Kanno A, Kubota K, Hirano K, Isayama H, Shimizu K, Tsuyuguchi T, Shimosegawa T, Kawa S, Chiba T, Okazaki K, Takikawa H, Kimura W, Unno M, Yoshida M: Clinical practice guidelines for IgG4-related sclerosing cholangitis. J Hepatobiliary Pancreat Sci. 2019; 26: 9-42.

37) Hakuta R, Kogure H, Nakai Y, Hamada T, Noguchi K, Saito K, Saito T, Takahara N, Mizuno S, Yagioka H, Ito Y, Tada M, Isayama H, Koike K: Endoscopic papillary large balloon 
dilation without sphincterotomy for users of antithrombotic agents: A multicenter retrospective study. Dig Endosc. 2019; 31: 316-322.

38) Nakai $Y$, Kogure H, Isayama H, Koike K: Endoscopic ultrasound-guided pancreatic duct drainage. Saudi J Gastroenterol. 2019; 25: 210217.

39) Sano M, Ijichi H, Takahashi R, Miyabayashi K, Fujiwara H, Yamada T, Kato H, Nakatsuka T, Tanaka Y, Tateishi K, Morishita Y, Moses HL, Isayama H, Koike K: Blocking CXCLs-CXCR2 axis in tumor-stromal interactions contributes to survival in a mouse model of pancreatic ductal adenocarcinoma through reduced cell invasion/migration and a shift of immune-inflammatory microenvironment. Oncogenesis. 2019; 8: 8.

40) Sasaki T, Sato T, Nakai Y, Sasahira N, Isayama $\mathrm{H}$, Koike K: Brain metastasis in pancreatic cancer: Two case reports. Medicine (Baltimore). 2019; 98: e14227.

41) Fujisawa T, Saito H, Isayama $H$ : Endoscopic removal of a metal stent that migrated into the peritoneal cavity after endoscopic ultrasoundguided hepaticogastrostomy. Dig Endosc. 2019; 31: e74-e75.

42) Kawaji Y, Isayama H, Nakai Y, Saito K, Sato T, Hakuta R, Saito T, Takahara N, Mizuno S, Kogure H, Matsubara S, Tada M, Kitano M, Koike K: Multiple recurrences after endoscopic removal of common bile duct stones: A retrospective analysis of 976 cases. J Gastroenterol Hepatol. 2019; 34: 1460-1466.

43) Hakuta R, Hamada T, Nakai Y, Isayama H, Koike K: Pancreatic stent during biliary cannulation: How can we catch 2 hares? Gastrointest Endosc. 2019; 89: 648-649.

44) Hamada $T$, Isayama $H$, Nakai $Y$, Iwashita $T$, Ito Y, Mukai T, Yagioka H, Saito T, Togawa O, Ryozawa S, Hirano K, Mizuno S, Yamamoto N, Kogure H, Yasuda I, Koike K: Antireflux covered metal stent for nonresectable distal malignant biliary obstruction: Multicenter randomized controlled trial. Dig Endosc, 2019; 31: 566-574.

45) Nakai Y, Kogure H, Isayama H, Koike K: Endoscopic Ultrasound-Guided Biliary Drainage for Benign Biliary Diseases. Clin Endosc, 2019; 52: 212-219.
46) Isayama H, Nakai $Y$, Itoi T, Yasuda I, Kawakami H, Ryozawa S, Kitano M, Irisawa A, Katanuma A, Hara K, Iwashita T, Fujita N, Yamao K, Yoshida M, Inui K: Clinical practice guidelines for safe performance of endoscopic ultrasound/ultrasonography-guided biliary drainage: 2018. J Hepatobiliary Pancreat Sci. 2019; 26: 249-269.

47) Saito T, Nakai Y, Mizuno S, Isayama H, Sasahira N, Watanabe T, Matsubara S, Arizumi T, Togawa O, Hirano K, Tsujino T, Ishigaki K, Hakuta R, Saito K, Takahara N, Hamada T, Kogure H, Tada M, Koike K: A randomized-controlled trial of early endotherapy versus wait-and-see policy for mild symptomatic pancreatic stones in chronic pancreatitis. Eur J Gastroenterol Hepatol. 2019; 31: 979-984.

48) Takahara N, Isayama H, Nakai Y, Sasaki T, Saito K, Sato T, Hakuta R, Ishigaki K, Saito T, Hamada T, Mizuno S, Kogure H, Tada M, Koike K: A feasibility study of gemcitabine, S-1 and leucovorin combination therapy (GSL) for advanced biliary tract cancer. J Chemother. 2019; 31: 284-289.

49) Nishiyama M, Ishii S, Fujisawa T, Saito H, Isayama $\mathrm{H}$ : Endoscopic removal of a migrated plastic stent from the peritoneal cavity after an EUS-guided gallbladder drainage procedure. VideoGIE. 2019; 4: 266-268.

50) Sato $T$, Kogure $H$, Nakai $Y$, Ishigaki $K$, Hakuta R, Saito K, Saito T, Takahara N, Hamada T, Mizuno S, Yamada A, Tada M, Isayama H, Koike K: Double-balloon endoscopy-assisted treatment of hepaticojejunostomy anastomotic strictures and predictive factors for treatment success. Surg Endosc, 2019 Jun 19. [Epub ahead of print]

51) Gonoi W, Okuma H, Hayashi TY, Akahane M, Nakai Y, Tateishi R, Mizuno S, Suzuki Y, Mitsuda M, Matsuda K, Nakagawa K, Isayama H, Miyagawa K, Koike K, Abe O: Development of pancreatic cancer during observation for hepatocellular carcinoma: A retrospective cohort study. Saudi J Gastroenterol. 2019; 25: 390-396.

52) Seo DW, Sherman S, Dua KS, Slivka A, Roy A, Costamagna G, Deviere J, Peetermans J, Rousseau M, Nakai Y, Isayama H, Kozarek R: 
Biliary SEMS During Neoadjuvant Therapy Study Group. Covered and uncovered biliary metal stents provide similar relief of biliary obstruction during neoadjuvant therapy in pancreatic cancer: a randomized trial. Gastrointest Endosc. 2019 Oct; 90: 602-612.e4.

53) Mizuno S, Isayama H, Nakai $Y$, Ishigaki K, Saito K, Sato T, Takeda T, Hakuta R, Saito T, Takahara N, Kogure H, Ijichi H, Tateishi K, Tada M, Shikata N, Tagami T, Kikuchi S, Yamamoto H, Yamakado M, Koike K: Diagnostic yield of the plasma free amino acid index for pancreatic cancer in patients with diabetes mellitus. Pancreatology. 2019; 19: 695-698.

54) Sato T, Kogure H, Nakai Y, Hamada T, Takahara N, Mizuno S, Kawaguchi Y, Akamatsu N, Kaneko J, Hasegawa K, Tada M, Tsujino T, Isayama H, Koike K: Long-term outcomes of endoscopic treatment for duct-to-duct anastomotic strictures after living donor liver transplantation. Liver Int. 2019; 39: 1954-1963.

55) Sato T, Nakai Y, Kogure H, Isayama H, Koike $\mathrm{K}$ : Electrohydraulic lithotripsy through a fistula of EUS-guided hepaticogastrostomy: a new approach for right intrahepatic stones. VideoGIE. 2019; 4: 420-422.

56) Nakai $Y$, Sato T, Hakuta R, Ishigaki K, Saito K, Saito T, Takahara N, Hamada T, Mizuno S, Kogure H, Tada M, Isayama H, Koike K: Management of Difficult Bile Duct Stones by Large Balloon, Cholangioscopy, Enteroscopy and Endosonography. Gut Liver, 2019 Oct 8. [Epub ahead of print]

57) Nakai $Y$, Isayama $H$, Wang HP, Rerknimitr $R$, Khor C, Yasuda I, Kogure H, Moon JH, Lau J, Lakhtakia S, Ratanachu-Ek T, Seo DW, Lee DK, Makmun D, Dy F, Liao WC, Draganov PV, Almadi M, Irisawa A, Katanuma A, Kitano M, Ryozawa S, Fujisawa T, Wallace MB, Itoi $\mathrm{T}$, Devereaux B: International consensus statements for endoscopic management of distal biliary stricture. J Gastroenterol Hepatol, 2019 Dec 4. [Epub ahead of print]

58) Fujisawa T, Isayama $H$, Gunji $T$, Sato $H$, Matsuhashi N: Prevalence Rate and Predictive Factors of Pancreatic Diseases in Cases with Pancreatic Duct Dilatation: A Cross-sectional Study of a Large, Healthy Japanese
Population. Intern Med, 2019 Dec 6. [Epub ahead of print]

59) Fujiwara H, Tateishi K, Misumi K, Hayashi A, Igarashi K, Kato H, Nakatsuka T, Suzuki N, Yamamoto K, Kudo Y, Hayakawa Y, Nakagawa H, Tanaka Y, Ijichi H, Kogure H, Nakai Y, Isayama H, Hasegawa K, Fukayama M, Soga T, Koike K: Mutant IDH1 confers resistance to energy stress in normal biliary cells through PFKP-induced aerobic glycolysis and AMPK activation. Sci Rep, 2019; 9: 18859 .

60) Takasaki Y, Irisawa A, Shibukawa G, Sato A, Abe Y, Yamabe A, Arakawa N, Maki T, Yoshida Y, Igarashi R, Yamamoto S, Ikeda T: New endoscopic ultrasonography criteria for malignant lymphadenopathy based on interrater agreement. PLoS One, 2019; 14: e0212427.

61) Yamabe A, Irisawa A, Bhutani MS, Sato A, Maki T, Takasaki Y, Yoshida Y, Yamamoto S, Shibukawa G: Validity of Endoscopic Ultrasound Findings of Chronic Pancreatitis: Evaluation from the Viewpoint of Disease Risk Factors. Digestion. 2019 Dec 4; 1-9. 2

62) Sato S, Sato S, Tsuzura H, et al: Elevated Serum Procalcitonin Levels and Their Association With the Prognosis of Patients With Liver Cirrhosis. [published online ahead of print, 2019 Dec 16]. Eur J Gastroenterol Hepatol.

$\langle$ Reviews〉

1) Kokudo N, Takemura N, Hasegawa K, Takayama T, Kubo S, Shimada M, Nagano H, Hatano E, Izumi N, Kaneko S, Kudo M, Iijima H, Genda T, Tateishi R, Torimura T, Igaki H, Kobayashi S, Sakurai H, Murakami T, Watadani T, Matsuyama Y: Clinical practice guidelines for hepatocellular carcinoma: The Japan Society of Hepatology 2017 (4th JSH-HCC guidelines) 2019 update. Hepatol Res. 2019 Oct; 49: 1109-1113.

2) Sakamoto N, Osada T, Nagahara A: Advanced Endoscopic Treatment for Early Colorectal Cancers. Juntendo Medical Journal. 2019; 65: 426-432.

3) Murakami T, Sakamoto N, Nagahara A: Clinicopathological features, diagnosis, and treatment of sessile serrated adenoma/polyp with dysplasia/carcinoma. J Gastroenterol Hepatol. 
2019; 34: 1685-1695.

\section{Cardiovascular Medicine}

〈Original Articles〉

1) Takamura K, Fujimoto S, Kawaguchi $Y$, Kato E, Aoshima C, Hiki M, Kumamaru KK, Daida $\mathrm{H}$ : The usefulness of low radiation dose subtraction coronary computed tomography angiography for patients with calcification using 320-row area detector CT. J Cardiol. 2019; 73: 58-64.

2) Fujimoto S, Kawasaki T, Kumamaru KK, Kawaguchi Y, Dohi T, Okonogi T, Ri K, Yamada S, Takamura K, Kato E, Kato Y, Hiki M, Okazaki S, Aoki S, Mitsouras D, Rybicki FJ, Daida H: Diagnostic performance of on-site computed CT-fractional flow reserve based on fluid structure interactions: comparison with invasive fractional flow reserve and instantaneous wave-free ratio. Eur Heart J Cardiovasc Imaging. 2019; 20: 343-352.

3) Kumamaru KK, Angel E, Sommer KN, Iyer V, Wilson MF, Agrawal N, Bhardwaj A, Kattel SB, Kondziela S, Malhotra S, Manion C, Pogorzelski K, Ramanan T, Sawant AC, Suplicki MM, Waheed S, Fujimoto S, Sharma UC, Rybicki FJ, Ionita C: Comprehensive analysis of inter- and intra-operator variability in measurement of on-site CT-derived fractional flow reserve based on structural and fluid analysis. Radiology: cardiothoracic imaging. 2019; https://doi.org/10.1148/ryct.2019180012.

4) Tomizawa N, Chou S, Matsuoka S, Yamamoto K, Inoh S, Nojo T, Kumamaru KK, Fujimoto S, Nakamura S: Optimizing contrast medium injection for coronary CT angiography using myocardial CT perfusion data. CVIA. 2019; 3: 106-112.

5) Shimizu T, Suwa S, Dohi T, Wada H, Miyauchi K, Shitara J, Endo H, Doi S, Ogita M, Kasai T, Okazaki S, Isoda K, Daida H: Clinical Significance of High-Sensitivity C-Reactive Protein in Patients with Preserved Renal Function Following Percutaneous Coronary Intervention. Int Heart J. 2019 Sep. 27; 60: 1037-1042.

6) Endo H, Dohi T, Dohi S, Wada H, Doi S, Kato Y, Okai I, Iwata H, Okazaki S, Isoda K, Yamamoto T, Miyauchi K, Amano A, Daida H: Clinical indicators and coronary angiographic features of expansive arterial remodelling in patients with abdominal aortic aneurysms. PLoS One. 2019 Jul 16; 14: e0219730.

7) Minami-Takano A, Iwata H, Miyosawa K, Kubota K, Kimura A, Osawa S, Shitara M, Okazaki S, Suwa S, Miyauchi K, Sumiyoshi M, Amano A, Daida H: A Novel Nutritional Index Serves as A Useful Prognostic Indicator in Cardiac Critical Patients Requiring Mechanical Circulatory Support. Nutrients. 2019 Jun 24; 11.

8) Inoue O, Osada M, Nakamura J, Kazama F, Shirai T, Tsukiji N, Sasaki T, Yokomichi H, Dohi T, Kaneko M, Kurano M, Oosawa M, Tamura S, Satoh K, Takano K, Miyauchi K, Daida H, Yatomi Y, Ozaki Y, Suzuki-Inoue K: Soluble CLEC-2 is generated independently of ADAM10 and is increased in plasma in acute coronary syndrome: comparison with soluble GPVI. Int J Hematol. 2019 Sep; 110: 285-294.

9) Wada H, Dohi T, Miyauchi K, Takahashi N, Endo H, Kato Y, Ogita M, Okai I, Iwata H, Okazaki S, Isoda K, Shimada K, Suwa S, Daida H: Impact of serum 1,5-anhydro-D-glucitol level on the prediction of severe coronary artery calcification: an intravascular ultrasound study.Cardiovasc Diabetol. 2019 Jun 3; 18: 69.

10) Aikawa T, Shimada K, Miyauchi K, Miyazaki T, Sai E, Ouchi S, Kadoguchi T, Kunimoto M, Joki Y, Dohi T, Okazaki S, Isoda K, Ohashi K, Murohara T, Ouchi N, Daida H: Associations among circulating levels of follistatin-like 1, clinical parameters, and cardiovascular events in patients undergoing elective percutaneous coronary intervention with drug-eluting stents. PLoS One. 2019 Apr 29; 14: e0216297.

11) Jujo K, Otsuki H, Tanaka K, Fukushima N, Okai I, Nakashima M, Dohi T, Okazaki S, Okabe R, Nagura F, Nara Y, Kawashima H, Kyono H, Arashi H, Yamaguchi J, Tamura H, Kurata T, Miyauchi K, Kozuma K, Daida H, Hagiwara N: Long-term cardiovascular prognosis after rotational atherectomy in hemodialysis patients: Data from the J2T multicenter registry. Int J Cardiol. 2019 Jun 15; 285: 14-20.

12) Yatsu S, Kasai T, Suda S, Matsumoto H, Ishiwata S, Shiroshita N, Kato M, Kawana F, 
Murata A, Shimizu M, Shitara J, Kato T, Hiki M, Sai E, Miyauchi K, Daida H: Prevalence and Significance of Restless Legs Syndrome in Patients With Coronary Artery Disease. Am J Cardiol. 2019 May 15; 123: 1580-1586.

13) Endo H, Dohi T, Miyauchi K, Kuramitsu S, Kato Y, Okai I, Yokoyama M, Yokoyama T, Ando K, Okazaki S, Shimada K, Suwa S, Daida H: Clinical significance of non-culprit plaque regression following acute coronary syndrome: A serial intravascular ultrasound study. J Cardiol. 2019 Aug; 74: 102-108.

14) Wada H, Miyauchi K, Daida H: J Cardiol. 2019 Aug; 74: 102-108. Expert Rev Cardiovasc Ther. 2019 Feb; 17: 127-133.

15) Endo H, Dohi T, Funamizu T, Shitara J, Wada H, Doi S, Naito R, Konishi H, Ogita M, Iwata H, Kasai T, Okazaki S, Isoda K, Shimada K, Miyauchi K, Daida H: Long-Term Predictive Value of High-Sensitivity C-Reactive Protein for Cancer Mortality in Patients Undergoing Percutaneous Coronary Intervention. Circ J. 2019 Feb 25; 83: 630-636.

16) Wada H, Dohi T, Miyauchi K, Daida H: Reply to: "Comment on "Mean platelet volume and long-term cardiovascular outcomes in patients with stable coronary artery disease"'. Atherosclerosis. 2019 Jan; 280: 195-196.

17) Dohi T, Kasai T, Endo H, Wada H, Yanagisawa N, Nojiri S, Funamizu T, Shitara J, Doi S, Kato Y, Okai I, Iwata H, Isoda K, Okazaki S, Miyauchi K, Daida H: CPAP effects on atherosclerotic plaques in patients with sleepdisordered breathing and coronary artery disease: The ENTERPRISE trial. J Cardiol. 2019 Jan; 73: 89-93.

18) Shitara J, Ogita M, Wada H, Tsuboi S, Endo H, Doi S, Konishi H, Naito R, Dohi T, Kasai T, Okazaki S, Isoda K, Suwa S, Miyauchi K, Daida H: Clinical impact of high-sensitivity C-reactive protein during follow-up on longterm adverse clinical outcomes in patients with coronary artery disease treated with percutaneous coronary intervention. J Cardiol. 2019 Jan; 73: 45-50.

19) Yasuda S, Kaikita K, Akao M, Ako J, Matoba T, Nakamura M, Miyauchi K, Hagiwara N, Kimura K, Hirayama A, Matsui K, Ogawa H: AFIRE Investigators. Antithrombotic Therapy for Atrial Fibrillation with Stable Coronary Disease. N Engl J Med. 2019 Sep 19; 381: 11031113.

20) Ako J, Hibi K, Tsujita K, Hiro T, Morino Y, Kozuma K, Shinke T, Otake H, Uno K, Louie MJ, Takagi Y, Miyauchi K: Effect of Alirocumab on Coronary Atheroma Volume in Japanese Patients With Acute Coronary Syndrome - The ODYSSEY J-IVUS Trial. Circ J. 2019 Sep 25; 83: 2025-2033.

21) Kimura K, Kimura T, Ishihara M, Nakagawa Y, Nakao K, Miyauchi K, Sakamoto T, Tsujita K, Hagiwara N, Miyazaki S, Ako J, Arai H, Ishii H, Origuchi $\mathrm{H}$, Shimizu W, Takemura $\mathrm{H}$, Tahara Y, Morino Y, Iino K, Itoh T, Iwanaga Y, Uchida K, Endo H, Kongoji K, Sakamoto K, Shiomi H, Shimohama T, Suzuki A, Takahashi J, Takeuchi I, Tanaka A, Tamura T, Nakashima T, Noguchi T, Fukamachi D, Mizuno T, Yamaguchi J, Yodogawa K, Kosuge M, Kohsaka S, Yoshino H, Yasuda S, Shimokawa H, Hirayama A, Akasaka T, Haze K, Ogawa H, Tsutsui H, Yamazaki T: Japanese Circulation Society Joint Working Group. JCS 2018 Guideline on Diagnosis and Treatment of Acute Coronary Syndrome.Circ J. 2019 Apr 25; 83: 1085-1196.

22) Matsumura-Nakano Y, Shizuta S, Komasa A, Morimoto T, Masuda H, Shiomi H, Goto K, Nakai K, Ogawa H, Kobori A, Kono Y, Kaitani K, Suwa S, Aoyama T, Takahashi M, Sasaki Y, Onishi Y, Mano T, Matsuda M, Motooka M, Tomita H, Inoko M, Wakeyama T, Hagiwara N, Tanabe K, Akao M, Miyauchi K, Yajima J, Hanaoka K, Morino Y, Ando K, Furukawa Y, Nakagawa Y, Nakao K, Kozuma K, Kadota K, Kimura K, Kawai K, Ueno T, Okumura K, Kimura T: OAC-ALONE Study Investigators.Open-Label Randomized Trial Comparing Oral Anticoagulation With and Without Single Antiplatelet Therapy in Patients With Atrial Fibrillation and Stable Coronary Artery Disease Beyond 1 Year After Coronary Stent Implantation. Circulation. 2019 Jan 29; 139: 604-616.

23) Kurokawa K, Sai E, Hayashi E, Minowa K, Sugano K, Yoshihara T, Miyazaki T, Hiki M, Yokoyama T, Suzuki M, Miyauchi K: Usefulness of Cardiac Magnetic Resonance in the 
Diagnosis of Löffler Endocarditis Secondary to Eosinophilic Granulomatosis with Polyangiitis. Intern Med. 2019 Jan 15; 58: 239-242.

24) Kawashima H, Kyono H, Nakashima M, Okai I, Jujo K, Dohi T, Otsuki H, Tanaka K, Nagura F, Okazaki S, Hagiwara N, Daida H, Kozuma K: Prognostic impact of scoring balloon angioplasty after rotational atherectomy in heavily calcified lesions using second-generation drugeluting stents: A multicenter registry-based study. Cardiovasc Revasc Med. 2019 May 23.

25) Kawaguchi YO, Fujimoto S, Kumamaru KK, Kato E, Dohi T, Takamura K, Aoshima C, Kamo Y, Kato Y, Hiki M, Okai I, Okazaki S, Aoki S, Daida H: The predictive factors affecting false positive in on-site operated CT-fractional flow reserve based on fluid and structural interaction.Int J Cardiol Heart Vasc. 2019 May 11; 23: 100372.

26) Saji M, Tobaru T, Higuchi R, Mahara K, Takamisawa I, Iguchi N, Doi S, Okazaki S, Tamura H, Takanashi S, Takayama M, Isobe M: Usefulness of the Transcatheter Aortic Valve Replacement Risk Score to Determine Mid-Term Outcomes. Circ J. 2019 Jul 25; 83: 1755-1761.

27) Yokoyama H, Tobaru T, Muto Y, Hagiya K, Higuchi R, Saji M, Takamisawa I, Shimizu J, Takanashi S, Takayama M, Tomita H, Tamura H, Doi S, Okazaki S, Isobe M: Long-term outcomes in Japanese nonagenarians undergoing transcatheter aortic valve implantation: A multi-center analysis. Clin Cardiol. 2019 Jun; 42: 605-611.

28) Kitamura K, Isoda K, Akita K, Miyosawa K, Kadoguchi T, Shimada K, Daida H: Lack of $\mathrm{I} \kappa \mathrm{BNS}$ promotes cholate-containing high-fat diet-induced inflammation and atherogenesis in low-density lipoprotein (LDL) receptordeficient mice.Int J Cardiol Heart Vasc. 2019 Mar 27; 23: 100344.

29) Terayama $M$, Yamada $K$, Hagiwara $T$, Inazuka F, Sezaki T, Igari T, Yokoi C, Nohara K, Soma D, Dohi T, Kawamura YI: Glutathione S-transferase omega 2 regulates cell growth and the expression of $\mathrm{E}$-cadherin via post-transcriptional downregulation of $\beta$-catenin in human esophageal squamous cells. Carcinogenesis, 18 November 2019.
30) Ujiie H, Iwata H, Yamagami J, Nakama T, Aoyama Y, Ikeda S, Ishii N, Iwatsuki K, Kurosawa M, Sawamura D, Tanikawa A, Tsuruta D, Nishie W, Fujimoto W, Amagai M, Shimizu $\mathrm{H}$ : Committee for Guidelines for the Management of Pemphigoid Diseases (Including Epidermolysis Bullosa Acquisita). Japanese guidelines for the management of pemphigoid (including epidermolysis bullosa acquisita). J Dermatol. 2019 Dec; 46: 1102-1135.

31) Yamamoto S, Iwata A, Yano Y, Ohmine T, Honma K, Senzaki K, Fujiwara M, Murakami T, Inoue J, Sano Y, Okagawa S, Otsuki Y, Wanaka H, Kataoka M, Iwata H: Preliminary study on the effects of movement velocity training of the upper limbs on gait ability in older adults: a nonrandomized controlled trial. Clin Interv Aging. 2019 May 1; 14: 781-788.

32) Iwata A, Sano Y, Wanaka H, Yamamoto S, Yano Y, Iwata H: Different improvement trends in gait function and quadriceps strength early after total knee arthroplasty. J Phys Ther Sci. 2019 Jan; 31: 57-62.

33) Aikawa T, Shimada K, Miyauchi K, Miyazaki T, Sai E, Ouchi S, Kadoguchi T, Kunimoto M, Joki Y, Dohi T, Okazaki S, Isoda K, Ohashi K, Murohara T, Ouchi N, Daida H: Associations among circulating levels of follistatin-like 1 , clinical parameters, and cardiovascular events in patients undergoing elective percutaneous coronary intervention with drug-eluting stents. PLoS One, 2019; 14: e0216297.

34) Ohmura H: Triglycerides as Residual Risk for Atherosclerotic Cardiovascular Disease. Circ J. 2019; 83: 969-970.

35) Ohmura H, Mita T, Matsuoka J, Nojiri S, Nishizaki Y, Watada H, Daida H, on behalf of SPIRITS-J Study Investigators. Real-World Data on the Incidence of Macrovascular Complications in Japanese Patients with Type 2 Diabetes: The Sitagliptin Registration Type 2 Diabetes-Juntendo Collaborating Project. Diabetes Ther 2019; 10: 1099-1111.

36) Daida H, Dohi T, Fukushima Y, Ohmura H, Miyauchi K: The goal of achieving atherosclerotic plaque regression with lipid-lowering therapy: Insights from IVUS trials. J Atheroscler Thromb 2019; 26: 592-600.

37) Daida H, Fukushima Y, Ohmura H: The Role 
of Aggressive Lipid Lowering Therapy for Coronary Artery Disease. Juntendo Medical Journal. 2019; 65: 468-473.

38) Perger E, Lyons OD, Inami T, Smith S, Floras JS, Logan AG, Bradley TD; ADVENT-HF Investigators; ADVENT-HF Trial Executive Committee:; ADVENT-HF Steering Committee:; ADVENT-HF Data and Safety Monitoring Committee:; ADVENT-HF Event Adjudication Committee:; ADVENT-HF Investigators:. Predictors of 1-year compliance with adaptive servoventilation in patients with heart failure and sleep disordered breathing: preliminary data from the ADVENT-HF trial. Eur Respir J. 2019; 53: 1801626.

39) Inami $T$, Kasai $T$, Yumino D, Perger E, Alshaer H, Hummel R, Lyons OD, Floras JS, Bradley TD*: Relationship of stroke volume to different patterns of Cheyne-Stokes respiration in patients with heart failure. Sleep. 2019; 42: zsy262.

40) Nishimura A, Kasai T, Kikuno S, Nagasawa K, Okubo M, Narui K, Mori Y: Apnea hypopnea index during rapid eye movement sleep with diabetic retinopathy in patients with type 2 diabetes. J Clin Endocrinol Metab. 2019; 104: 2075-2082.

41) Yatsu $S$, Kasai $T^{*}$, Matsumoto $H$, Shitara J, Shimizu M, Murata A, Kato T, Suda S, Hiki M, Takagi A, Daida H: Relationship between hypoalbuminemia on admission and longterm mortality in patients with acute decompensated heart failure. Intern Med. 2019; 58: 1695-1702.

* 42) Shitara J, Kasai T*, Konishi H, Endo H, Wada H, Doi S, Naito R, Tsuboi S, Ogita M, Dohi T, Okazaki S, Miyauchi K, Daida H: Impact of lipoprotein (a) levels on long-term outcomes in patients with coronary artery disease and left ventricular systolic dysfunction. Circ J. 2019; 83: 1047-1053.

* 43) Matsumoto H, Kasai T*, Sato A, Ishiwata S, Yatsu S, Shitara J, Murata A, Takao Kato T, Suda S, Matsue Y, Hiki M, Takagi A, Daida $\mathrm{H}$ : Association between $\mathrm{C}$-reactive protein level at hospital admission and long-term mortality in patients with acute decompensated heart failure. Heart Vessels. 2019; 34: 1961-1968.
44) Kikuchi T, Kasai $\mathrm{T}^{*}$, Tomita $\mathrm{Y}$, Kimura $\mathrm{Y}$, Miura J, Tamura H, Mitani H, Narui K, Ishiwata S: Relationship between sleep disordered breathing and heart rate turbulence in non-obese subjects. Heart Vessels. 2019; 34: 1801-1810.

* 45) Tomita Y, Kasai T*, Ishiwata S, Daida H, Narui K: Aortic Knob Width as a Parameter of atherosclerosis in patients with obstructive sleep apnea. J Atheroscler Thromb. 2019 Sep 28. [Epub ahead of print]

* 46) Kawasaki Y, Kasai T*, Koikawa N, Hanazato N, Suda S, Murata A, Ozaki R, Nagai S, Matsumura Y, Kaneko H, Kubo M, Osawa A, Nojiri S, Ogasawara E, Sakuraba K, Daida H, Kitade M, Itakura A: Sex differences in factors associated with poor subjective sleep quality in athletes. J Sports Med Phys Fitness. 2019 Oct 16. [Epub ahead of print]

47) Murase K, Tanizawa K, Minami T, Matsumoto T, Tachikawa R, Takahashi N, Tsuda T, Ohi M, Akahoshi T, Tomita Y, Narui K, Nakamura H, Ohdaira T, Yoshimine H, Tsuboi T, Yamashiro Y, Ando S, Kasai T, Kita H, Tatsumi K, Burioka N, Tomii K, Kondo Y, Takeyama H, Handa T, Hamada S, Oga T, Nakayama T, Sakamaki T, Morita S, Kuroda T, Hirai T, Chin K*: Telemedicine for obstructive sleep apnea patients undergoing long-term CPAP treatment. Ann Am Thorac Soc. 2019 Nov 5. [Epub ahead of print]

48) Nojiri $S^{*}$, Itoh H, Kasai T, Fujibayashi K, Saito T, Hiratsuka Y, Okuzawa A, Naito T, Yokoyama $\mathrm{K}$, Daida H: Comorbidity status in hospitalized elderly in Japan: analysis from national database of health insurance claims and specific health checkups. Sci Rep. 2019; 9: 20237.

49) Kimura $Y$, Kasai $T^{*}$, Tomita $Y$, Kasagi S, Takaya H, Kato M, Kawana F, Narui K: Relationship between metabolic syndrome and hypercapnia among obese patients with sleep apnea. World J Respirol. 2019; 10: 1-10.

50) Kaya E, Miyazaki S, Kaneko T, Morimoto R, Maruyama M, Hirose K, Daida H: The Prevalence of the Double-Barrel View with Prominent Eustachian Valve in Patients with Atrial Septal Defect. J Am Soc Echocardiogr. 2019 Dec; 32: 1618-1619.

51) Watanabe H, Domei T, Morimoto T, Natsuaki 
M, Shiomi H, Toyota T, Ohya M, Suwa S, Takagi K, Nanasato M, Hata Y, Yagi M, Suematsu N, Yokomatsu Y, Takamisawa I, Doi M, Noda T, Okayama H, Seino Y, Tada T, Sakamoto H, Hibi K, Abe M, Kawai K, Nakao K, Ando K, Tanabe K, Ikari Y, Hanaoka KI, Morino Y, Kozuma K, Kadota K, Furukawa Y, Nakagawa Y, Kimura K: Effect of 1-month dual antiplatelet therapy followed by clopidogrel vs 12 -month dual antiplatelet therapy on cardiovascular and bleeding events in patients receiving PCI: The STOP DAPT-2 randomized clinical trial.JAMA. 2019 Jun 25; 321: 2414-2427.

52) Wada H, Miyauchi K, Daida H: Gender differences in the clinical features and outcomes of patients with coronary artery disease. Expert Rev Cardiovasc Ther. 2019 Feb; 17.

53) Shiomi H, Kozuma K, Morimoto T, Kadota K, Tanabe K, Morino Y, Akasaka T, Abe M, Takeji Y, Suwa S, Ito Y, Kobayashi M, Dai K, Nakao K, Tarutani Y, Taniguchi R, Nishikawa H, Yamamoto Y, Nakagawa Y, Ando K, Kobayashi K, Kawai K, Hibi K, Kimura T: 7year outcomes of a randomized trial comparing the first-generation sirolimus-eluting stent versus the new generation everolimuseluting stent: the RESET trial. JACC Cardiovasc Interv. 2019 Apr; 12; 637-647.

54) Okuno T, Aoki J, Tanabe K, Nakao K, Ozaki Y, Kimura K, Ako J, Noguchi T, Yasuda S, Suwa S, Fujimoto K, Nakama Y, Morita T, Shimizu W, Saito Y, Hirohata A, Morita Y, Inoue $\mathrm{T}$, Okamura $\mathrm{A}$, Uematsu M, Hirata K, Shibata Y, Owa M, Tsujita K, Funayama H, Kokubu N, Kozuma K, Uemura S, Toubaru T, Saku K, Ohshima S, Nakai M, Nishimura K, Miyamoto Y, Ogawa H, Ishihara M: Admission heart rate is a determinant of effectiveness of beta-blockers in acute myocardial infarction patients. Circ J. 2019 Apr; 83: 10541063.

55) Okura H, Saito Y, Soeda T, Nakao K, Ozaki Y, Kimura K, Ako J, Noguchi T, Yasuda S, Suwa S, Fujimoto K, Nakama Y, Morita T, Shimizu W, Saito Y, Hirohata A, Morita Y, Inoue T, Okamura A, Uematsu M, Hirata K, Tanabe K, Shibata Y, Owa M, Tsujita K, Funayama H, Kokubu N, Kozuma K, Uemura S, Toubaru T,
Saku K, Ohshima S, Nakai M, Nishimura K, Miyamoto Y, Ogawa H, Ishihara M: Frequency and prognostic impact of intravascular imaging-guided urgent percutaneous coronary intervention in patients with acute myocardial infarction: results from J-MINUET. Heart Vessels. 2019 Apr; 34: 564-571.

56) Ueki $Y$, Mohri M, Matoba T, Kadokami T, Suwa S, Yagi T, Takahashi H, Takana N, Hokama Y, Fukuhara R, Onitsuka K, Tachibana E, Yonemoto N, Nagao K: Prognostic value of neurological status on hospital arrival for short-term outcome in patients with cardiovascular shock - sub-analysis of the Japanese Circulation Society Cardiovascular Shock RegistryCirc J. 2019 May 24; 83: 12471253.

57) Yanagawa Y, Jitsuiki K, Nagasawa H, Takeuchi I, Madokoro S, Ohsaka H, Ishikawa K, Omori K, Suwa S: A smartphone video transmission system for verification of transfusion.Air Med J. 2019 May-Apr; 38: 125-128.

58) Endo H, Miyauchi K, Takahashi D, Funamizu T, Shitara J, Wada H, Doi S, Kato Y, Okai I, Iwata H, Okazaki S, Isoda K, Daida H: Clinical impact of complex percutaneous coronary intervention in patients with coronary artery disease. Cardiovasc Interv Ther. 2019 Jul 26. doi:10.1007/s12928-019-00608-7.〔Epub ahead of print]

59) Ueki Y, Mohri M, Matoba T, Kadokami T, Suwa S, Yagi T, Takahashi H, Takana N, Hokama Y, Fukuhara R, Onitsuka K, Tachibana E, Yonemoto N, Nagao K: Clinical characteristics and prognostic factors in acute coronary syndrome patients complicated with cardiogenic shock in Japan: analysis from the Japanese Circulation Society Cardiovascular Shock Registry. Heart vessels. 2019 Aug; 34: 1241-1249.

60) Wada H, Dohi T, Miyauchi K, Takahashi N, Endo H, Kato Y, Ogita M, Okai I, Iwata H, Okazaki S, Isoda K, Shimada K, Suwa S, Daida H: Impact of sesrum 1,5-anhydro-Dglucitol level on the prediction of severe coronary artery calcification: an intravascular ultrasound study.Int Heart J. 2019 Sep 27; 60: 1037-1042.

61) Yatsu S, Kasai T, Daida H: Reply to Comments 
on Relationship between Hypoalbuminemia on Admission and Long-term Mortality in Patients with Acute Decompensated Heart Failure. Intern Med. 2019 Dec 1; 58: 3491.

62) Kunimoto M, Shimada K, Yokoyama M, Matsubara T, Aikawa T, Ouchi S, Shimizu M, Fukao K, Miyazaki T, Kadoguchi T, Fujiwara K, Honzawa A, Yamada M, Shimada A, Yamamoto T, Amano A, Daida H: Relationship Between the Kihon Checklist and the Clinical Parameters in Patients Who Participated in Cardiac Rehabilitation. Geriatr Gerontol Int. 2019; 19: 287-292.

63) Matsushita K, Harada K, Miyazaki T, Miyamoto T, Kohsaka S, Iida K, Yamamoto Y, Nagatomo Y, Yoshino H, Yamamoto T, Nagao K, Takayama M: Younger- Vs Older-Old Patients With Heart Failure With Preserved Ejection Fraction. J Am Geriatr Soc. 2019; 67: 2123-2128.

64) Tokano T, Nakazato Y: The Combination of a Basket Catheter and EnSite Mapping System Improves Catheter Ablation of Right Ventricle Outflow Premature Ventricular Contraction. Edited by Mohammad Shenasa, Gerhard Hindricks, David J. Callans, John Miller, and Mark E. Josephson. 5th edition of Cardiac Mapping. Wiley Blackwell. March 2019.

65) Aikawa T, Shimada K, Miyauchi K, Miyazaki T, Sai E, Ouchi S, Kadoguchi T, Kunimoto M, Joki Y, Dohi T, Okazaki S, Isoda K, Ohashi K, Murohara T, Ouchi N, Daida H: Associations among circulating levels of follistatin-like 1, clinical parameters, and cardiovascular events in patients undergoing elective percutaneous coronary intervention with drug-eluting stents. PLoS One. 2019 Apr 29; 14: e0216297.

66) Kadoguchi T, Shimada K, Miyazaki T, Kitamura K, Kunimoto M, Aikawa T, Sugita Y, Ouchi S, Shiozawa T, Yokoyama-Nishitani M, Fukao K, Miyosawa K, Isoda K, Daida H: Promotion of oxidative stress associates with mitochondrial dysfunction and muscle atrophy in aging mice. Geriatr Gerontol Int. 2019 in press.

67) Tadaki S, Sakata Y, Miura Y, Nochioka K, Miura M, Miyata S, Asakura M, Shimada K, Yamamoto T, Fukumoto Y, Kadokami T, Yasuda S, Miura T, Ando S, Yano M, Kita- kaze M, Daida H, Shimokawa H: Factors Alienating Habitual Exercise in Patients with Chronic Heart Failure "A Multicenter Prospective Cohort Study”. Heart Vessels. 2019 in press.

68) Someya Y, Tamura Y, Kaga H, Nojiri S, Shimada K, Daida H, Ishijima M, Kaneko K, Aoki S, Miida T, Hirayama S, Konishi S, Hattori N, Motoi Y, Naito H, Kawamori R, Watada H: Cohort profile: skeletal muscle function and need for long-term care in a prospective cohort study of urban elderly people in Japan (the Bunkyo Health Study). BMJ Open. 2019 Sep 17; 9: e031584.

69) Kaga H, Tamura Y, Takeno K, Kakehi S, Someya Y, Funayama T, Furukawa M, Suzuki R, Sugimoto D, Kadowaki S, Nishitani-Yokoyama M, Shimada K, Daida H, Aoki S, Giacca A, Kanazawa A, Kawamori R, Watada $\mathrm{H}$ : Higher C-peptide level during Glucose Clamp is Associated with Muscle Insulin Resistance in Non-obese Japanese Men. J Endocr Soc. 2019 Jul 23; 3: 1847-1857.

70) Kadowaki S, Tamura Y, Someya Y, Takeno K, Kaga H, Sugimoto D, Kakehi S, Funayama T, Furukawa M, Suzuki R, Nishitani-Yokoyama M, Shimada K, Daida H, Aoki S, Kanazawa A, Kawamori R, Watada H: Fatty liver has stronger association with insulin resistance than visceral fat accumulation in non-obese Japanese men. J Endocr Soc. 2019 May 20; 3: 1409-1416.

71) Li M, Hirano KI, Ikeda Y, Higashi M, Hashimoto C, Zhang B, Kozawa J, Sugimura K, Miyauchi H, Suzuki A, Hara Y, Takagi A, Ikeda Y, Kobayashi K, Futsukaichi Y, Zaima N, Yamaguchi S, Rojeet S, Nakamura H, Kawaguchi K, Sai E, Hui SP, Nakano Y, Sawamura A, Inaba T, Sakata Y, Yasui Y, Nagasawa Y, Kinugawa S, Shimada K, Yamada S, Hao H, Nakatani D, Ide T, Amano T, Naito H, Nagasaka $\mathrm{H}$, Kobayashi $\mathrm{K}$ on behalf of the Japan TGCV study group: Triglyceride deposit cardiomyovasculopathy: a rare cardiovascular disorder. Orphanet J Rare Dis. 2019 Jun 11; 14: 134.

72) Sugimoto D, Tamura Y, Takeno K, Kaga H, Someya Y, Kakehi S, Funayama T, Furukawa Y, Suzuki R, Kadowaki S, Nishitani-Yo- 
koyama M, Shimada K, Daida H, Aoki S, Kanazawa A, Kawamori R, Watada H: Clinical features of non-obese, apparently healthy Japanese men with reduced adipose tissue insulin sensitivity. J Clin Endocrinol Metab. 2019; 104: 2325-2333.

73) Yatsu S, Kasai T, Suda S, Matsumoto H, Ishiwata S, Shiroshita N, Kato M, Kawana F, Murata A, Shimizu M, Shitara J, Kato T, Hiki M, Sai E, Miyaucji K, Daida H: Prevalence and Significance of Restless Legs Syndrome in Patients With Coronary Artery Disease. Am J Cardiol. 2019 Feb 23.

74) Yatsu S, Kasai T, Matsumoto H, Shitara J, Shimizu M, Murata A, Kato T, Suda S, Hiki M, Takagi A, Daida H: Relationship between Hypoalbuminemia on Admission and Longterm Mortality in Patients with Acute Decompensated Heart Failure. Intern Med. 2019 Feb 25; 1716-18.

75) Shitara J, Kasai T, Akihiro S, Yatsu S, Matsumoto H, Suda S, Ogita M, Yanagisawa N, Fujibayashi K, Nojiri S, Nishizaki Y, Ono N, Suwa S, Daida H: Effects of suvorexant on sleep apnea in patients with heart failure: A protocol of crossover pilot trial. J Cardiol. 2019 Jul; 74: 90-94.

76) Yatsu S, Kasai T, Suda S, Hiki M, Matsumoto H, Ishiwata S, Sato A, Shiroshita N, Kato M, Kawana F, Murata A, Shimizu M, Shitara J, Kato T, Sai E, Yanagisawa N, Miyauchi K, Daida H: Prevalence of Restless Legs Syndrome and Its Effects on Sleep and Health-Related Quality of Life in Patients With Heart Failure. J Card Fail. 2019 Oct; 25: 837-842.

77) Kitai T, Xanthopoulos A, Tang WHW, Kaji S, Furukawa Y, Oishi S, Akiyama E, Suzuki S, Yamamoto M, Kida K, Okumura T, Skoulargis J, Triposkiadis F, Matsue Y: Validation of the Larissa Heart Failure Risk Score for Risk Stratification in Acute Heart Failure. Int J Cardiol 2019; in-press.

78) Matsue Y. Are obese patients with heart failure very different from non-obese patients? J Card Fail 2019; in-press.

79) Matsue Y, Kagiyama N, Yamaguchi T, Kuroda S, Okumura T, Kida K, Mizuno A, Oishi S, Inuzuka Y, Akiyama E, Matsukawa R, Koto K, Yoshioka K, Miyoshi T, Baba Y, Yama- moto M, Mizutani K, Yoshida K, Kitai T: Clinical and prognostic values of ALBI score in patients with acute heart failure. Heart Lung Circ 2019; in-press.

80) Yoshioka K, Matsue Y*, Yamaguchi T, Kitai T, Kagiyama N, Okumura T, Kida K, Oishi S, Akiyama E, Suzuki S, Yamamoto M, Kuroda S, Matsumura A, Hirao K: Safety and prognostic impact of early treatment with angiotensin converting enzyme inhibitor or angiotensin receptor blocker in patients with acute heart failure. Am J Cardiovasc Drugs. 2019; 19: 597-605.

81) Kagiyama N, Kitai T, Hayashida A, Yamaguchi T, Okumura T, Kida K, Mizuno A, Oishi S, Inuzuka Y, Akiyama E, Suzuki S, Yamamoto M, Shimizu A, Urakami Y, Toki M, Aritaka S, Matsumoto K, Nagano N, Yamamoto K, Matsue Y*: Prognostic Value of BNP Reduction during Hospitalization in Patients with Acute Heart Failure. J Card Fail. 2019; 25: 712-721.

82) Hattori M, Naruse Y, Oginosawa Y, Matsue Y, Hanaki Y, Kowase S, Kurosaki K, Mizukami A, Kohno R, Abe H, Aonuma K, Nogami A: Prognostic Impact of Lead Tip Position Confirmed via Computed Tomography in Patients with Right Ventricular Septal Pacing. Heart Rhythm. 2019; 16: 921-927.

83) Saito H, Matsue $Y^{*}$, Suzuki M, Kamiya K, Hasegawa Y, Endo Y, Negishi Y, Hirano M, Takanashi K, Iizuka H, Matsumura A, Hashimoto Y: Discordance between subjective and objective evaluations of cognitive function in old Japanese patients with heart failure. Australas J Ageing. 2019; 38: 57-59.

84) Saito H, Kagiyama N, Nagano N, Matsumoto K, Yoshioka K, Endo Y, Hayashida A, Matsue $\mathrm{Y}$ : Social isolation is associated with 90 -day rehospitalization due to heart failure. Eur J Cardiovasc Nurs. 2019; 18: 16-20.

85) Nozaki K, Kamiya K, Matsue Y, Hamazaki N, Matsuzawa R, Tanaka S, Maekawa E, Kishi T, Matsunaga A, Masuda T, Izumi T, Ako J: Pupillary light reflex as a new prognostic marker in patients with heart failure. J Card Fail. 2019; 25: 156-163.

86) Kuroda S, Damman K, ter Maaten JM, Voors AA, Okumrua T, Kida K, Oishi S, Akiyama E, 
Suzuki S, Yamamoto M, Kitai T, Yoshida K, Matsumura A, Matsue Y*: Very early diuretic response after admission for acute heart failure. J Card Fail. 2019; 25: 12-19.

〈Reviews〉

1) Daida H, Dohi T, Fukushima Y, Ohmura H, Miyauchi K: The Goal of Achieving Atherosclerotic Plaque Regression with Lipid-Lowering Therapy: Insights from IVUS Trials. J Atheroscler Thromb. 2019 Jul 1; 26: 592-600.

2) Murata A, Kasai T*: Treatment of central sleep apnea in patients with heart failure: Now and future. World J Respirol. 2019; 9: 1-7.

3) Fukuda K*, Date H, Doi S, Fukumoto Y, Fukushima N, Hatano M, Ito H, Kuwana M, Matsubara H, Momomura SI, Nishimura M, Ogino H, Satoh T, Shimokawa H, Yamauchi-Takihara K, Tatsumi K, Ishibashi-Ueda H, Yamada N, Yoshida S, Abe K, Ogawa A, Ogo T, Kasai T, Kataoka M, Kawakami T, Kogaki S, Nakamura M, Nakayama T, Nishizaki M, Sugimura K, Tanabe N, Tsujino I, Yao A, Akasaka T, Ando M, Kimura T, Kuriyama T, Nakanishi N, Nakanishi T, Tsutsui $\mathrm{H}$ : Japanese Circulation Society and the Japanese Pulmonary Circulation and Pulmonary Hypertension Society Joint Working Group. Guidelines for the Treatment of Pulmonary Hypertension (JCS 2017/JPCPHS 2017). Circ J. 2019; 83: 842-945.

4) Inoshita $A^{*}$, Kasai $T$, Matsuoka $R$, Sata $N$, Shiroshita N, Kawana F, Kato M, Ikeda K: Sex differences in the development of upper airway morphology: is this the new kid on the block? J Thorac Dis. 2019; 11 (Suppl 15): S2032-S2033.

5) Kwon $Y^{*}$, Logan J, Pusalavidyasagar S, Kasai T, Cheong CSJ, Lee CH: Sleep apnea and heart. Sleep Med Res. 2019; 10: 67-74.

6) Tsutsui $\mathrm{H}$, Isobe $\mathrm{M}$, Ito $\mathrm{H}$, Ito $\mathrm{H}$, Okumura $\mathrm{K}$, Ono M, Kitakaze M, Kinugawa K, Kihara Y, Goto Y, Komuro I, Saiki Y, Saito Y, Sakata Y, Sato N, Sawa Y, Shiose A, Shimizu W, Shimokawa H, Seino Y, Node K, Higo T, Hirayama A, Makaya M, Masuyama T, Murohara T, Momomura SI, Yano M, Yamazaki K,
Yamamoto K, Yoshikawa T, Yoshimura M, Akiyama M, Anzai T, Ishihara S, Inomata T, Imamura T, Iwasaki YK, Ohtani T, Onishi K, Kasai T, Kato M, Kawai M, Kinugasa Y, Kinugawa S, Kuratani T, Kobayashi S, Sakata Y, Tanaka A, Toda K, Noda T, Nochioka K, Hatano M, Hidaka T, Fujino T, Makita S, Yamaguchi O, Ikeda U, Kimura T, Kohsaka S, Kosuge M, Yamagishi M, Yamashina A: Japanese Circulation Society and the Japanese Heart Failure Society Joint Working Group. JCS 2017/JHFS 2017 Guideline on diagnosis and treatment of acute and chronic heart failure- digest version. Circ J. 2019; 83: 2084-2184.

7) Negishi T, Miyazaki S, Negishi K: Echocardiography and Cardio-Oncology. Heart Lung Circ. 2019 Sep; 28: 1331-1338.

8) Tokano T, Nakazato Y: Edited by Mohammad Shenasa, Gerhard Hindricks, David J. Callans, John Miller, and Mark E. Josephson. 5th edition of Cardiac Mapping. Wiley Blackwell. March 2019.

9) Shimada K, Nishitani-Yokoyama M, Takahashi T, Daida H: Physical activity and longterm prognosis in patients with stable coronary artery disease: how often, how intense, and how long? Eur J Prev Cardiol. 2019 in press.

10) Shimada K, Fukushima Y, Fukao K, Shiozaki M, Hiki M, Aihara K, Daida H: The Tokyo 2020 Olympic and Paralympic Games and Sportology: a comprehensive approach for preventing cardiovascular diseases during the Olympic and Paralympic Games. Juntendo Medical Journal. 2019 in press.

$\langle$ Books $\rangle$

1) Tokano T, Nakazato Y: The Combination of a Basket Catheter and EnSite Mapping System Improves Catheter Ablation of Right Ventricle Outflow Premature Ventricular Contraction. Edited by Mohammad Shenasa, Gerhard Hindricks, David J. Callans, John Miller, and Mark E. Josephson. 5th edition of Cardiac Mapping. Wiley Blackwell. March 2019. 